\title{
On the Complexity of Pure-Strategy Nash Equilibria in Congestion and Local-Effect Games
}

\author{
Juliane Dunkel \\ Operations Research Center, Massachusetts Institute of Technology, Cambridge, Massachusetts 02139, \\ juliane@mit.edu \\ Andreas S. Schulz \\ Sloan School of Management, Massachusetts Institute of Technology, Cambridge, Massachusetts 02139, \\ schulz@mit.edu
}

\begin{abstract}
Rosenthal's congestion games constitute one of the few known classes of noncooperative games possessing pure-strategy Nash equilibria. In the network version, each player wants to route one unit of flow on a single path from her origin to her destination at minimum cost, and the cost of using an arc depends only on the total number of players using that arc. A natural extension is to allow for players controlling different amounts of flow, which results in so-called weighted congestion games. While examples have been exhibited showing that pure-strategy Nash equilibria need not exist anymore, we prove that it is actually strongly NP-hard to determine whether a given weighted network congestion game has a purestrategy Nash equilibrium. This is true regardless of whether flow is unsplittable or not. In the unsplittable case, the problem remains strongly NP-hard for a fixed number of players. In addition to congestion games, we provide complexity results on the existence and computability of pure-strategy Nash equilibria for the closely related family of bidirectional local-effect games. Therein, the cost of a player taking a particular action depends not only on the number of players choosing the same action, but also on the number of players settling for (locally) related actions.
\end{abstract}

Key words: noncooperative games; pure-strategy Nash equilibria; computational complexity; congestion games; local-effect games

MSC2000 subject classification: Primary: 90B10, 90B20, 91A10; secondary: 90C27, 91A43

OR/MS subject classification: Primary: networks/graphs: multicommodity, theory; secondary: games: noncooperative; mathematics: combinatorics

History: Received May 21, 2007; revised December 20, 2007. Published online in Articles in Advance October 17, 2008.

1. Introduction. Game theory in general, and the concept of Nash equilibrium in particular, has lately (re)emerged as a "hot topic" in the operations research and computer science literature. The complexity of computing a mixed Nash equilibrium of a finite game given in strategic form is a case in point. Goldberg and Papadimitriou [20] showed that finding a mixed Nash equilibrium in a game with a constant number of players can be reduced to solving a four-player game. Daskalakis et al. [10] showed in turn that the latter problem is PPAD-complete, i.e., it is as difficult as computing a Brouwer fix point of a continuous function from the closed unit ball to itself. Subsequently, Chen and Deng [7] and Daskalakis and Papadimitriou [9] proved that computing mixed Nash equilibria in games with three players is PPAD-complete as well. Eventually, Chen and Deng [8] established the same result for the two-player case.

Although Nash [30] showed that mixed Nash equilibria do exist in any finite noncooperative game, it is well known that pure-strategy Nash equilibria may not, as demonstrated by classical games such as "matching pennies" (e.g., Shor [35]). It is therefore natural to ask which games have pure-strategy Nash equilibria and, if applicable, how difficult it is to find one. In this article, we study these questions for certain classes of weighted congestion games and local-effect games.

Congestion games were introduced by Rosenthal [31], who proved that they are guaranteed to possess purestrategy Nash equilibria. In fact, Monderer and Shapley [29] showed that every exact potential game is isomorphic to a congestion game. In a congestion game, a player's strategy consists of a subset of resources, and her cost depends only on the number of players choosing the same resources. An important subclass of congestion games can be represented by means of networks. ${ }^{1}$ Every player wants to route one unit of flow from her origin to her destination, on a path of minimal cost. The network arcs are the resources, and a player's set of pure strategies consists of the sets of arcs corresponding to paths connecting her origin-destination pair. Fabrikant et al. [12] studied the computational complexity of finding pure-strategy Nash equilibria in congestion games. For symmetric network congestion games, where all players have the same origin-destination pair, they presented a polynomial-time algorithm for computing a pure-strategy Nash equilibrium. On the other hand, they proved that this problem is PLS-complete for symmetric congestion games as well as for asymmetric network

${ }^{1}$ Network congestion games are particularly interesting from a computational point of view because players' strategies can be encoded compactly. 
congestion games. A simpler proof of the latter result was given by Ackermann et al. [1], who also showed that this result still holds for affine cost functions.

In weighted congestion games, players control different, integral amounts of flow. Depending on whether players are allowed to split their flows or not, a player's strategy consists of a set of paths with corresponding integer flow values between her origin-destination pair, or a single path. Libman and Orda [25] constructed a simple instance of an unsplittable weighted network congestion game that does not possess a pure-strategy Nash equilibrium. A similar example was presented by Fotakis et al. [16], who also observed that for the special case of affine cost functions, a pure-strategy Nash equilibrium is always guaranteed to exist. Awerbuch et al. [5] derived a tight bound of $(\sqrt{5}+3) / 2$ on the pure price of anarchy for this special case. The pure price of anarchy is the ratio of the cost of a worst pure-strategy Nash equilibrium to that of a globally optimal solution. Awerbuch et al. also gave upper bounds for instances with polynomial cost functions of degree greater than one. Tight bounds for this case were later provided by Aland et al. [3]. Goemans et al. [19] showed that a pure-strategy Nash equilibrium need not exist for instances with cost functions that are polynomials of degree of at most two. Milchtaich [27] had earlier shown that weighted congestion games with player-specific cost functions on networks consisting only of parallel arcs do not always have a pure-strategy Nash equilibrium either. This was elaborated on by Gairing et al. [17], who considered different cost functions, slight modifications in the network topology, and both the weighted and the unweighted cases. Milchtaich [28] characterized topological properties of networks that guarantee the existence of pure-strategy Nash equilibria in network congestion games if players control different amounts of flow or cost functions are player specific.

In this article, we prove that the problem of deciding whether a weighted network congestion game with simple, nonlinear cost functions possesses a pure-strategy Nash equilibrium is strongly NP-hard, regardless of whether one considers splittable or unsplittable flows. In the unsplittable case, we are able to show that the problem remains strongly NP-complete even if the number of players is fixed, or if all players have the same origin and destination. We also establish strong NP-completeness for weighted congestion games with affine player-specific cost functions on networks consisting of parallel arcs only.

Leyton-Brown and Tennenholtz [24] introduced local-effect games to model situations in which the use of one resource can affect the cost of using other resources. Local-effect games are, in general, not guaranteed to possess pure-strategy Nash equilibria. However, Leyton-Brown and Tennenholtz showed that so-called bidirectional local-effect games with linear local-effect functions belong to the class of exact potential games, and therefore always have pure-strategy Nash equilibria. The question of whether there exists a polynomial-time algorithm for finding a pure-strategy Nash equilibrium for these games was left open.

We prove that computing a pure-strategy Nash equilibrium is, in fact, PLS-complete. Because the proof uses a tight PLS-reduction, our result implies the existence of instances of bidirectional local-effect games with linear local-effect functions that have exponentially long shortest improvement paths. It also implies that the problem of computing a pure-strategy Nash equilibrium that is reachable from a given strategy state via selfish improvement steps is PSPACE-hard. In addition, we show that, given an initial strategy profile for a bidirectional local-effect game with linear local-effect functions and a positive integer $k$ (unarily encoded), it is strongly NP-complete to decide whether there is a sequence of at most $k$ selfish steps that transforms the initial state into a pure-strategy Nash equilibrium. We also prove that the problem of deciding whether a bidirectional local-effect game with general local-effect functions has a pure-strategy Nash equilibrium is strongly NP-complete.

Before we present the details of our results on weighted congestion games and local-effect games in $\S \S 3$ and 4 , respectively, we conclude this introduction by briefly discussing additional related work on the computational complexity of pure-strategy Nash equilibria. Gottlob et al. [21] considered restrictions of strategic games intended to capture certain aspects of bounded rationality. Among other results, they proved that even in the setting where each player's payoff function depends on the actions of at most three other players and where each player's strategy set consists of at most three actions, the problem of determining whether a strategic game has a purestrategy Nash equilibrium is NP-complete. This result was strengthened by Fischer et al. [13], who showed that the problem remains NP-hard even if each player has only two actions to choose from and her payoff depends on the actions of at most two other players. Àlvarez et al. [4] studied how various representations of a strategic game influence the computational complexity of deciding the existence of a pure-strategy Nash equilibrium. They showed that this problem is NP-complete when the number of players is large and the number of strategies for each player is constant, whereas the problem is $\sum_{2}^{p}$-complete when the number of players is constant and the sizes of the strategy sets are exponential (with respect to the lengths of the strategies). Schoenebeck and Vadhan [34] analyzed the computational complexity of deciding whether pure-strategy Nash equilibria exist in graph games and circuit games. Brandt et al. [6] studied the impact of various notions of symmetry in strategic games on the computational complexity of finding pure-strategy Nash equilibria. Expanding on a line of research 
started by Ieong et al. [22], who considered singleton congestion games, Ackermann et al. [1] proved that the lengths of all best-response sequences are polynomially bounded in the number of players and resources, in congestion games where the strategy space of each player consists of the bases of a matroid over the set of resources. This especially implies that pure-strategy Nash equilibria for congestion games with this matroid property can be computed in polynomial time, even in the case of player-specific costs (Ackermann et al. [2]). In the latter paper, Ackermann et al. also showed the existence of pure-strategy Nash equilibria in weighted congestion games with the same matroid property.

\section{Preliminaries.}

Noncooperative games. A strategic game is defined by a set $N$ of $n$ players, a finite set of actions $S_{i}$ for each player $i \in N$, and a payoff or utility function $u_{i}$ for each player mapping $S:=\prod_{i \in N} S_{i}$ to $\mathbb{Q}$. The set $S$ is called the strategy or action space of the game, and its elements are the pure-strategy states. A purestrategy Nash equilibrium of a strategic game is a state $s=\left(s_{1}, s_{2}, \ldots, s_{n}\right) \in S$ such that for each player $i \in N$, $u_{i}(s) \geq u_{i}\left(s_{1}, \ldots, s_{i-1}, s_{i}^{\prime}, s_{i+1}, \ldots, s_{n}\right)$, for all $s_{i}^{\prime} \in S_{i}$. Thus, no player can benefit from changing her strategy while the other players retain their current strategies.

Although every game has a Nash equilibrium if players are allowed to randomize over their set of pure actions (Nash [30]), pure-strategy Nash equilibria are, in general, not guaranteed to exist. A fundamental class of strategic games, which always have a pure-strategy Nash equilibrium, are potential games. Every game in this class is characterized by the existence of a potential function that associates with each strategy profile a real number such that the change in the potential function value of two states differing only in a single player's strategy is positive if and only if the difference in payoff to this particular player is positive. A potential function is exact if these two values always coincide.

Congestion games. An unweighted congestion game is defined by a set of players $N=\{1,2, \ldots, n\}$ and a set of resources $E$. For each player $i \in N$, her set of available strategies is a collection of subsets of the resources; i.e., $S_{i} \subseteq 2^{E}$. A nondecreasing cost function $f_{e}: \mathbb{N} \rightarrow \mathbb{Q}_{\geq 0}$ is associated with each resource $e \in E$. Given a strategy profile $s=\left(s_{1}, s_{2}, \ldots, s_{n}\right) \in S$, the cost of player $i$ is $c_{i}(s)=-u_{i}(s)=\sum_{e \in s_{i}} f_{e}\left(n_{e}(s)\right)$, where $n_{e}(s)$ denotes the number of players using resource $e$ in $s$. In other words, in a congestion game each player chooses a subset of resources that are available to her; and the cost to a player is the sum of the costs of the resources used by her, where the cost of a resource depends only on the total number of players sharing this resource.

A network congestion game is a congestion game in which the arcs of an underlying directed network represent the resources. Each player $i \in N$ has an origin-destination pair $\left(r_{i}, t_{i}\right)$, where $r_{i}$ and $t_{i}$ are nodes of the network, and the set $S_{i}$ of pure strategies available to player $i$ is the set of directed (simple) paths from $r_{i}$ to $t_{i}$.

In a weighted network congestion game, each player $i \in N$ has a positive integer weight $w_{i}$, which constitutes the amount of flow that player $i$ wants to ship from $r_{i}$ to $t_{i}$. In the case of unsplittable flows, the cost of player $i$ adopting strategy $s_{i}$ in a strategy profile $s=\left(s_{1}, s_{2}, \ldots, s_{n}\right) \in S$ is given by $c_{i}(s)=\sum_{e \in s_{i}} f_{e}\left(\theta_{e}(s)\right)$, where $\theta_{e}(s)=\sum_{i: e \in s_{i}} w_{i}$ denotes the total flow on arc $e$ in $s$. In integer-splittable network congestion games, a player with weight greater than one can choose a subset of paths on which to route her flow simultaneously; that is, player $i$ 's strategy consists of the specification of the $r_{i}-t_{i}$-paths used and the integer amounts of flow routed on them, which sum up to $w_{i}$. The corresponding cost is the total cost of the paths that player $i$ uses, weighted by the respective amounts of flow player $i$ routes on them.

An (un)weighted network congestion game is called symmetric or a single-commodity game if all players have the same origin-destination pair.

In terms of the input size of a weighted network congestion game, we assume that the cost functions are explicitly specified; that is, for each integer value $\theta$ with $0 \leq \theta \leq \sum_{i \in N} w_{i}$ and each arc $e$, the value $f_{e}(\theta)$ is given in binary encoding. ${ }^{2}$

Local-effect games. In a local-effect game with player set $N=\{1,2, \ldots, n\}$, all players have the same set of available actions, $\mathscr{A}$. For each action $a \in \mathscr{A}$, there is a nondecreasing cost function $f_{a}: \mathbb{N} \rightarrow \mathbb{Q}_{\geq 0}$ that depends merely on the number of players who play this action. Furthermore, for each pair of actions $a, a^{\prime} \in \mathscr{A}, a \neq a^{\prime}$, a function $f_{a^{\prime}, a}: \mathbb{N} \rightarrow \mathbb{Q}_{\geq 0}$ expresses the impact of action $a^{\prime}$ on the cost of action $a$. Its value depends only on the

${ }^{2}$ Although more compact encodings are often possible, this assumption leads to stronger hardness results, which are the main concern of this paper. 
number of players that choose action $a^{\prime}$. The functions $f_{a^{\prime}, a}$ are called local-effect functions, and it is assumed that $f_{a^{\prime}, a}(0)=0$. Moreover, local-effect functions are either strictly increasing or identical to zero. For a given strategy state $s=\left(s_{1}, s_{2}, \ldots, s_{n}\right) \in \mathscr{A}^{n}, n_{a}(s)$ denotes the number of players playing action $a$ in $s$. The cost to a player $i \in N$ for playing action $s_{i}$ in strategy state $s$ is given by $c_{i}(s)=f_{s_{i}}\left(n_{s_{i}}(s)\right)+\sum_{a \in S, a \neq s_{i}} f_{a, s_{i}}\left(n_{a}(s)\right)$. If the local-effect functions $f_{a^{\prime}, a}$ are zero for all $a \neq a^{\prime}$, the local-effect game is equivalent to a symmetric network congestion game with parallel arcs. A local-effect game is called a bidirectional local-effect game if, for all $a, a^{\prime} \in \mathscr{A}, a \neq a^{\prime}$, and for all $x \in \mathbb{N}, f_{a^{\prime}, a}(x)=f_{a, a^{\prime}}(x)$.

PLS. The complexity class $P L S$ was introduced by Johnson et al. [23] to characterize the computational complexity of local search problems. A combinatorial optimization problem $\Pi$ consists of a collection of instances $(\mathscr{F}, c)$, where $\mathscr{F}$ denotes the set of feasible solutions and $c: \mathscr{F} \rightarrow \mathbb{Q}$ is the objective function. A combinatorial optimization problem $\Pi$ together with a neighborhood function $N: \mathscr{F} \rightarrow 2^{\mathscr{F}}$ belongs to PLS if (a) instances are recognizable in polynomial time and a feasible solution can be computed efficiently, (b) the feasibility of a proposed solution can be checked efficiently and its objective function value can be evaluated in polynomial time, and (c) the neighborhood of a feasible solution can be searched in polynomial time to determine a better feasible solution, if one exists. The computational problem associated with a local search problem is to find, for a given instance $(\mathscr{F}, c)$, a locally optimal solution w.r.t. the neighborhood function $N$, i.e., an $s \in \mathscr{F}$ such that there is no solution in the neighborhood of $s$ with strictly better cost. A local search problem $L_{2}$ in PLS is PLS-complete if, for any other problem $L_{1}$ in PLS, there are polynomial-time computable functions $\phi$ and $\psi$ such that $\phi$ maps instances $x$ of $L_{1}$ to instances $\phi(x)$ of $L_{2}, \psi$ maps solutions of $\phi(x)$ to solutions of $x$, and if $s$ is a locally optimal solution for the instance $\phi(x)$ of $L_{2}$, then $\psi(s, x)$ is a locally optimal solution for $x$. Such a reduction is called tight if for any instance $x$ of $L_{1}$ one can identify a subset $X$ of feasible solutions of $\phi(x)$ so that (a) $X$ contains all local optima of $\phi(x)$, (b) for every solution $f$ of $x$ one can construct in polynomial time a solution $s \in X$ such that $\psi(s, x)=f$, and (c) if the transition graph of $\phi(x)$ contains a directed path from $s \in X$ to $s^{\prime} \in X$ whose internal nodes are not in $X$, then either $\psi(s, x)=\psi\left(s^{\prime}, x\right)$ or the transition graph of $x$ contains an arc from $\psi(s, x)$ to $\psi\left(s^{\prime}, x\right)$ (Schäffer and Yannakakis [33]). In particular, the length of a longest path from any solution to a locally optimal solution in the transition graph of $L_{2}$ is at least as large as that in the transition graph of $L_{1}$.

3. Complexity of weighted congestion games. We begin by giving a high-level description of the common idea that forms the basis of our NP-hardness proofs for the various classes of games. In each case, we take a counterexample, i.e., an instance that does not have a pure-strategy Nash equilibrium, and couple it with an instance of the same class in which the strategy profiles correspond to the feasible solutions in a given instance of an NP-complete problem. We also introduce an additional player who can participate in either game. All other players are limited by cost or structure to participate in "their" part of the game only. The participation of the extra player in the counterexample turns that game into one that has a pure-strategy Nash equilibrium. Therefore, the entire game has a pure-strategy Nash equilibrium if and only if the part of the game corresponding to the NP-complete problem has a pure-strategy Nash equilibrium that prevents the extra player from joining the game, which happens if and only if it corresponds to a YES-instance. The only deviation from this proof scheme occurs in the case of weighted network congestion games with a fixed number of players. Instead of introducing another player, one of the two players from the counterexample is given access to the other part of the game, which she will be able to take advantage of if and only if the state in this part of the game corresponds to a solution of a YES-instance.

3.1. Unsplittable flows. Libman and Orda [25] presented an example of a weighted network congestion game with general nondecreasing arc-cost functions that does not have a pure-strategy Nash equilibrium. Fotakis et al. [16] provided a similar instance. We simplify the latter instance and turn it into a gadget to derive the following result.

THEOREM 3.1. The problem of deciding whether a weighted symmetric network congestion game with unsplittable flows possesses a pure-strategy Nash equilibrium is strongly NP-complete.

Proof. The proof is by reduction from 3-Partition, which is strongly NP-complete (Garey and Johnson [18]). Consider an arbitrary instance of 3-Partition: a finite set $A=\{1,2, \ldots, 3 m\}$ of items $(m \geq 2)$, a number $B \in \mathbb{N}$, and a positive integer weight $w_{i}$ for each item $i \in A$ such that $B / 4<w_{i}<B / 2$ and $\sum_{i \in A} w_{i}=m B$. We will construct a weighted single-commodity network congestion game that has a pure-strategy Nash equilibrium if and only if $A$ can be partitioned into $m$ disjoint sets $A_{1}, A_{2}, \ldots, A_{m}$ such that $\sum_{i \in A_{k}} w_{i}=B$ for $1 \leq k \leq m$. 


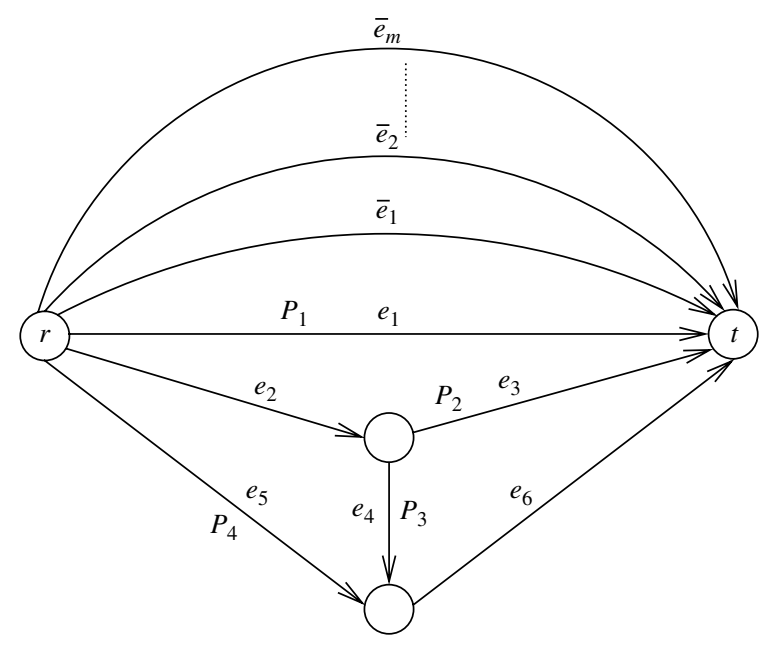

FigURE 1. Illustration of the weighted single-commodity network congestion game used in the proof of Theorem 3.1.

We introduce a player $p_{i}$ for each item $i \in A$; the corresponding weight is $w_{i}$. In addition, there are three players $p_{3 m+1}, p_{3 m+2}$, and $p_{3 m+3}$ with weights $w_{3 m+1}=\bar{B}, w_{3 m+2}=2 \bar{B}$, and $w_{3 m+3}=\bar{B} / 2$, respectively. Here, $\bar{B}:=2 m B$. All players have the same origin, $r$, and destination, $t$. The network is depicted in Figure 1. It consists of a contracted version of the example by Fotakis et al. [16] and $m$ additional arcs $\bar{e}_{1}, \bar{e}_{2}, \ldots, \bar{e}_{m}$, connecting $r$ and $t$. We denote the $r$ - $t$-paths in the lower part of the network by $P_{1}=\left(e_{1}\right), P_{2}=\left(e_{2}, e_{3}\right), P_{3}=\left(e_{2}, e_{4}, e_{6}\right)$, and $P_{4}=\left(e_{5}, e_{6}\right)$.

The nondecreasing arc-cost functions are defined as follows:

$$
\begin{aligned}
& f_{\bar{e}_{k}}(x):=\left\{\begin{array}{ll}
x, & \text { if } x<(m+1) B, \\
240 \bar{B}, & \text { otherwise, }
\end{array} \text { for } k=1, \ldots, m,\right. \\
& f_{e_{1}}(x):= \begin{cases}12 \bar{B}, & \text { if } x \leq \bar{B}, \\
120 \bar{B}, & \text { if } \bar{B}<x \leq 2 \bar{B}, \\
228 \bar{B}, & \text { otherwise, }\end{cases} \\
& f_{e_{2}}(x):= \begin{cases}1 \bar{B}, & \text { if } x \leq \bar{B}, \\
2 \bar{B}, & \text { if } \bar{B}<x \leq 2 \bar{B}, \\
8 \bar{B}, & \text { otherwise, }\end{cases} \\
& f_{e_{3}}(x):= \begin{cases}16 \bar{B}, & \text { if } x \leq \bar{B}, \\
18 \bar{B}, & \text { if } \bar{B}<x \leq 2 \bar{B}, \\
20 \bar{B}, & \text { otherwise, }\end{cases} \\
& f_{e_{4}}(x):= \begin{cases}1 \bar{B}, & \text { if } x \leq \bar{B}, \\
40 \bar{B}, & \text { if } \bar{B}<x \leq 2 \bar{B}, \\
79 \bar{B}, & \text { otherwise, }\end{cases} \\
& f_{e_{5}}(x):= \begin{cases}10 \bar{B}, & \text { if } x \leq \bar{B}, \\
12 \bar{B}, & \text { if } \bar{B}<x \leq 2 \bar{B}, \\
14 \bar{B}, & \text { otherwise, }\end{cases} \\
& f_{e_{6}}(x):= \begin{cases}2 \bar{B}, & \text { if } x \leq \bar{B}, \\
10 \bar{B}, & \text { if } \bar{B}<x \leq 2 \bar{B}, \\
12 \bar{B}, & \text { otherwise. }\end{cases}
\end{aligned}
$$


TABLE 1. Possible defections in the subgame defined by the subnetwork of the network displayed in Figure 1 that consists of $\operatorname{arcs} e_{1}, e_{2}, \ldots, e_{6}$ and players $p_{3 m+1}$ and $p_{3 m+2}$ only.

\begin{tabular}{|c|c|c|c|c|}
\hline$s_{3 m+1}$ & $s_{3 m+2}$ & Deviator $\rightarrow$ New strategy & Current $\operatorname{cost} / \bar{B}$ & Improved cost $/ \bar{B}$ \\
\hline$P_{1}$ & $P_{1}$ & $p_{3 m+1} \rightarrow P_{3}$ & $c_{3 m+1}=228$ & $c_{3 m+1}^{\prime}=4$ \\
\hline$P_{1}$ & $P_{2}$ & $p_{3 m+1} \rightarrow P_{3}$ & $c_{3 m+1}=12$ & $c_{3 m+1}^{\prime}=11$ \\
\hline$P_{1}$ & $P_{3}$ & $p_{3 m+2} \rightarrow P_{2}$ & $c_{3 m+2}=52$ & $c_{3 m+2}^{\prime}=20$ \\
\hline$P_{1}$ & $P_{4}$ & $p_{3 m+2} \rightarrow P_{2}$ & $c_{3 m+2}=22$ & $c_{3 m+2}^{\prime}=20$ \\
\hline$P_{2}$ & $P_{1}$ & $p_{3 m+2} \rightarrow P_{2}$ & $c_{3 m+2}=120$ & $c_{3 m+2}^{\prime}=28$ \\
\hline$P_{2}$ & $P_{2}$ & $p_{3 m+1} \rightarrow P_{1}$ & $c_{3 m+1}=28$ & $c_{3 m+1}^{\prime}=12$ \\
\hline$P_{2}$ & $P_{3}$ & $p_{3 m+1} \rightarrow P_{1}$ & $c_{3 m+1}=24$ & $c_{3 m+1}^{\prime m+1}=12$ \\
\hline$P_{2}$ & $P_{4}$ & $p_{3 m+1} \rightarrow P_{1}$ & $c_{3 m+1}=17$ & $c_{3 m+1}^{\prime}=12$ \\
\hline$P_{3}$ & $P_{1}$ & $p_{3 m+2} \rightarrow P_{2}$ & $c_{3 m+2}=120$ & $c_{3 m+2}^{\prime}=26$ \\
\hline$P_{3}$ & $P_{2}$ & $p_{3 m+2} \rightarrow P_{4}$ & $c_{3 m+2}=26$ & $c_{3 m+2}^{\prime}=24$ \\
\hline$P_{3}$ & $P_{3}$ & $p_{3 m+1} \rightarrow P_{1}$ & $c_{3 m+1}=99$ & $c_{3 m+1}^{\prime}=12$ \\
\hline$P_{3}$ & $P_{4}$ & $p_{3 m+1} \rightarrow P_{1}$ & $c_{3 m+1}=14$ & $c_{3 m+1}^{\prime m+1}=12$ \\
\hline$P_{4}$ & $P_{1}$ & $p_{3 m+2} \rightarrow P_{2}$ & $c_{3 m+2}=120$ & $c_{3 m+2}^{\prime}=20$ \\
\hline$P_{4}$ & $P_{2}$ & $p_{3 m+1} \rightarrow P_{3}$ & $c_{3 m+1}=12$ & $c_{3 m+1}^{\prime}=11$ \\
\hline$P_{4}$ & $P_{3}$ & $p_{3 m+1} \rightarrow P_{1}$ & $c_{3 m+1}=22$ & $c_{3 m+1}^{\prime m+1}=12$ \\
\hline$P_{4}^{+}$ & $P_{4}$ & $p_{3 m+1} \rightarrow P_{1}$ & $c_{3 m+1}=26$ & $c_{3 m+1}^{\prime m+1}=12$ \\
\hline
\end{tabular}

Suppose there is a partition $A_{1}, A_{2}, \ldots, A_{m}$ of $A$ such that $\sum_{i \in A_{k}} w_{i}=B$ for $1 \leq k \leq m$. Consider the strategy state $s$ in which player $p_{i}$ chooses the arc $\bar{e}_{k}$ such that item $i \in A_{k}$, and $s_{3 m+1}=P_{3}, s_{3 m+2}=P_{4}$, and $s_{3 m+3}=P_{1}$. The cost to player $p_{i}$, for $i \in A$, is $B$. If such a player would route her flow on one of the other $\operatorname{arcs} \bar{e}_{\ell}, \ell \neq k$, her cost would increase to $B+w_{i}$. By choosing a path $P_{k}, k \in\{1,2,3,4\}$, this player's cost would increase to at least $4 \bar{B}$. Player $p_{3 m+1}$ experiences a cost of $14 \bar{B}$. A change to path $P_{1}, P_{2}$, or $P_{4}$ would result in a cost of $120 \bar{B}, 17 \bar{B}$, or $26 \bar{B}$. For player $p_{3 m+2}$, the cost in state $s$ is $24 \bar{B}$. Switching to path $P_{1}, P_{2}$, or $P_{3}$ increases her cost to $228 \bar{B}, 26 \bar{B}$, or $99 \bar{B}$. Player $p_{3 m+3}$ has a cost of $12 \bar{B}$. Routing her flow on path $P_{2}, P_{3}$, or $P_{4}$ would result in an increased cost of $18 \bar{B}, 54 \bar{B}$, or $26 \bar{B}$. Finally, every player $p_{3 m+1}, p_{3 m+2}$, and $p_{3 m+3}$ would increase her cost to $240 \bar{B}$ by switching to some single-arc path $\bar{e}_{k}, k \in\{1,2, \ldots, m\}$. Thus, no player can decrease her cost by routing flow over another path: $s$ is a pure-strategy Nash equilibrium.

For the other direction, we claim that every pure-strategy Nash equilibrium $s$ of this game has the following properties:

(a) Players $p_{3 m+1}, p_{3 m+2}$, and $p_{3 m+3}$ play a strategy in $\left\{P_{1}, P_{2}, P_{3}, P_{4}\right\}$.

(b) Every player $p_{i}, i \in\{1,2, \ldots, 3 m\}$, chooses an arc $\bar{e}_{k}$, for some $1 \leq k \leq m$.

Property (a) clearly holds for players $p_{3 m+1}$ and $p_{3 m+2}$, for if one of these players chose an arc $\bar{e}_{k}$ for some $k \in\{1,2, \ldots, m\}$, she would experience a cost of $240 \bar{B}$. She could decrease her cost to at most $228 \bar{B}$ by switching to some path $P_{k}, k \in\{1,2,3,4\}$. For property (b), suppose there is a player $p_{i}, i \in\{1,2, \ldots, 3 m\}$, routing her flow on a path $P_{k}, k \in\{1,2,3,4\}$. In this case, the cost of player $p_{i}$ is at least $4 \bar{B}$. Given that we have already established property (a) for players $p_{3 m+1}$ and $p_{3 m+2}$, the total weight of players using an arc $\bar{e}_{k}$ in $s$ is at most $2 m B$. Therefore, there must exist some $k \in\{1,2, \ldots, m\}$ such that $\sum_{i: s_{i}=\bar{e}_{k}} w_{i} \leq 2 B$. By switching to $\operatorname{arc} \bar{e}_{k}$, player $p_{i}$ can decrease her cost to, at most, $f_{\bar{e}_{k}}\left(2 B+w_{i}\right)<3 B$. To show (a) for player $p_{3 m+3}$, suppose that she uses an arc $\bar{e}_{k}, k \in\{1,2, \ldots, m\}$. Then, only players $p_{3 m+1}$ and $p_{3 m+2}$ use one of the paths $P_{1}, P_{2}, P_{3}$, and $P_{4}$. However, the congestion game restricted to players $p_{3 m+1}$ and $p_{3 m+2}$ and strategies $P_{1}, P_{2}, P_{3}$, and $P_{4}$ does not have a pure-strategy Nash equilibrium - a contradiction. ${ }^{3}$ Table 1 lists the 16 possible combinations that we need to consider to show that in each case at least one of the two players can decrease her cost by routing her flow on a different path. Hence, the only way that the entire game can have a pure-strategy Nash equilibrium is for player $p_{3 m+3}$ to play a strategy in $\left\{P_{1}, P_{2}, P_{3}, P_{4}\right\}$.

Given a pure-strategy Nash equilibrium $s$, we can now define a partition of $A$ by setting $A_{k}:=\left\{i \in A: s_{i}=\bar{e}_{k}\right\}$, $k=1,2, \ldots, m$. We claim that these sets define a solution to the 3-Partition problem. Suppose this is not the case. Then, because of (a), there exists an index $k \in\{1,2, \ldots, m\}$ such that $\sum_{i: s_{i}=\bar{e}_{k}} w_{i}<B$. The current cost of player $p_{3 m+3}$, using a path $P_{k}, k \in\{1,2,3,4\}$, is at least $4 \bar{B}$. By switching to arc $\bar{e}_{k}$, this player can decrease her cost to, at most, $f_{\bar{e}_{k}}(B-1+m B)=(m+1) B-1$. This contradicts the assumption of $s$ being a Nash equilibrium.

To complete the proof, we note that the problem of deciding whether a weighted network congestion game with unsplittable flows has a pure-strategy Nash equilibrium is in NP. Indeed, one can verify in polynomial time that a given strategy state is a Nash equilibrium by conducting a shortest-path computation for each player.

${ }^{3}$ This subgame coincides with the contracted version of the instance by Fotakis et al. [16], to which we referred earlier. 
While the NP-hardness of the corresponding decision problem for weighted network congestion games with player-specific payoff functions follows immediately, we can actually strengthen this result.

THEOREM 3.2. The problem of deciding whether a weighted network congestion game with parallel arcs and affine player-specific cost functions possesses a pure-strategy Nash equilibrium is strongly NP-complete.

Proof. The problem is obviously in NP. To show NP-completeness, we reduce, as before, from 3-PARTITION. We are given a set $A=\{1,2, \ldots, 3 m\}$ of items, a number $B \in \mathbb{N}$, and a positive integer weight $w_{i}$ for each item $i \in A$ such that $B / 4<w_{i}<B / 2$ and $\sum_{i \in A} w_{i}=m B$. We will construct a weighted network congestion game with parallel arcs only and player-specific cost functions such that it has a pure-strategy Nash equilibrium if and only if $A$ can be partitioned into $m$ disjoint sets $A_{1}, A_{2}, \ldots, A_{m}$ such that $\sum_{i \in A_{k}} w_{i}=B$, for $1 \leq k \leq m$.

We introduce a player $p_{i}$ for each item $i \in A$; the corresponding weight is $w_{i}$. There are four additional players $p_{3 m+i}$, for $i=1,2,3,4$, with $w_{3 m+1}=1, w_{3 m+2}=2, w_{3 m+3}=3$, and $w_{3 m+4}=1$. All players want to ship flow from $r$ to $t$ in a network of parallel arcs $e_{1}, e_{2}, \ldots, e_{m+3}$ connecting $r$ and $t$.

Let $f_{i, k}$ denote the cost function of player $p_{i}$ for arc $e_{k}$, and let $K:=3(m B+7)+1$. For $i=1,2, \ldots, 3 m$ and $k=1,2, \ldots, m+3$, we define

$$
f_{i, k}(x):= \begin{cases}x, & \text { if } k \in\{1,2, \ldots, m\} \\ K, & \text { otherwise. }\end{cases}
$$

For the remaining players $p_{3 m+i}, i \in\{1,2,3,4\}$, we set

$$
\begin{aligned}
& f_{3 m+1, k}(x):= \begin{cases}K, & \text { if } k \in\{1,2, \ldots, m+1\}, \\
7, & \text { if } k=m+2, \\
2 x, & \text { if } k=m+3,\end{cases} \\
& f_{3 m+2, k}(x):= \begin{cases}K, & \text { if } k \in\{1,2, \ldots, m+1\}, \\
2 x, & \text { if } k=m+2, \\
5, & \text { if } k=m+3,\end{cases} \\
& f_{3 m+3, k}(x):= \begin{cases}K, & \text { if } k \in\{1,2, \ldots, m\} \cup\{m+2\}, \\
3 x, & \text { if } k=m+1, \\
2 x, & \text { if } k=m+3,\end{cases} \\
& f_{3 m+4, k}(x):= \begin{cases}x, & \text { if } k \in\{1,2, \ldots, m\}, \\
K, & \text { if } k \in\{m+1, m+2\}, \\
B+1, & \text { if } k=m+3 .\end{cases}
\end{aligned}
$$

Assume that we are given a YES-instance of the partition problem. Then there is a partition $A_{1}, A_{2}, \ldots, A_{m}$ of $A$ such that $\sum_{i \in A_{k}} w_{i}=B$ for $1 \leq k \leq m$. Consider the strategy state $s$ in which player $p_{i}$ chooses the arc $e_{k}$ with $i \in A_{k}, i=1,2, \ldots, 3 m$, and $s_{3 m+1}=e_{m+3}, s_{3 m+2}=e_{m+2}, s_{3 m+3}=e_{m+1}$, and $s_{3 m+4}=e_{m+3}$. In $s$, each player $p_{i}$ corresponding to an item $i \in A$ has a cost of $B$. Switching to a different arc in $\left\{e_{1}, e_{2}, \ldots, e_{m}\right\}$ increases her cost to $B+w_{i}$, and routing her flow on an arc in the set $\left\{e_{m+1}, e_{m+2}, e_{m+3}\right\}$ results in a cost of $K$, which is no improvement either. Player $p_{3 m+1}$ has a cost of four. The only other arc yielding a cost less than $K$ is $e_{m+2}$. However, switching to this arc results in a higher cost of seven. Player $p_{3 m+2}$ has a cost of four in state $s$. Changing her strategy to $e_{m+3}$ gives a new cost of five; all other arcs have cost $K$ for this player. Player $p_{3 m+3}$ with current cost nine can decrease her cost neither by using arc $e_{m+3}$, which would yield a cost of 10 , nor by taking one of the other arcs, which would result in a cost of $K$. Player $p_{3 m+4}$ 's cost is $B+1$ in $s$. Switching to an $\operatorname{arc} e_{k}$, for some $1 \leq k \leq m$, results in the same cost; all other arcs would increase her cost. Hence, $s$ is a pure-strategy Nash equilibrium.

For the other direction of the proof, we first observe that any pure-strategy Nash equilibrium of the constructed game has the following properties:

(a) Each player $p_{i}, i \in\{1,2, \ldots, 3 m\}$, uses an arc in $\left\{e_{1}, e_{2}, \ldots, e_{m}\right\}$.

(b) None of the players $p_{3 m+1}, p_{3 m+2}, p_{3 m+3}$ plays a strategy in $\left\{e_{1}, e_{2}, \ldots, e_{m}\right\}$.

(c) Player $p_{3 m+4}$ plays strategy $e_{m+3}$. 
TABLE 2. Possible defections in the subgame restricted to players $p_{3 m+1}, p_{3 m+2}, p_{3 m+3}$ and strategies $e_{m+1}, e_{m+2}, e_{m+3}$, as discussed in the proof of Theorem 3.2. This game is similar to instances described by Milchtaich [27].

\begin{tabular}{|c|c|c|c|c|c|}
\hline$s_{3 m+1}$ & $s_{3 m+2}$ & $s_{3 m+3}$ & Defector $\rightarrow$ New strategy & Current cost & Improved cost \\
\hline$e_{m+2}$ & $e_{m+2}$ & $e_{m+1}$ & $p_{3 m+1} \rightarrow e_{m+3}$ & $c_{3 m+1}=7$ & $c_{3 m+1}^{\prime}=2$ \\
\hline$e_{m+2}$ & $e_{m+2}$ & $e_{m+3}$ & $p_{3 m+2} \rightarrow e_{m+3}$ & $c_{3 m+2}=6$ & $c_{3 m+2}^{\prime}=5$ \\
\hline$e_{m+2}$ & $e_{m+3}$ & $e_{m+1}$ & $p_{3 m+1} \rightarrow e_{m+3}$ & $c_{3 m+1}=7$ & $c_{3 m+1}^{\prime}=6$ \\
\hline$e_{m+2}$ & $e_{m+3}$ & $e_{m+3}$ & $p_{3 m+3} \rightarrow e_{m+1}$ & $c_{3 m+3}=10$ & $c_{3 m+3}^{\prime}=9$ \\
\hline$e_{m+3}$ & $e_{m+2}$ & $e_{m+1}$ & $p_{3 m+3} \rightarrow e_{m+3}$ & $c_{3 m+3}=9$ & $c_{3 m+3}^{\prime}=8$ \\
\hline$e_{m+3}$ & $e_{m+2}$ & $e_{m+3}$ & $p_{3 m+1} \rightarrow e_{m+2}$ & $c_{3 m+1}=8$ & $c_{3 m+1}^{\prime}=7$ \\
\hline$e_{m+3}$ & $e_{m+3}$ & $e_{m+1}$ & $p_{3 m+2} \rightarrow e_{m+2}$ & $c_{3 m+2}=5$ & $c_{3 m+2}^{\prime}=4$ \\
\hline$e_{m+3}$ & $e_{m+3}$ & $e_{m+3}$ & $p_{3 m+3} \rightarrow e_{m+1}$ & $c_{3 m+3}=12$ & $c_{3 m+3}^{\prime}=9$ \\
\hline
\end{tabular}

Properties (a) and (b) follow immediately from the fact that for any player there exists a strategy with cost strictly smaller than $K$, i.e., in any Nash equilibrium each player pays less than $K$. For property (c), we first observe that in any Nash equilibrium the only possible strategies for player $p_{3 m+4}$ are $e_{k}, 1 \leq k \leq m$, and $e_{m+3}$. Suppose that $p_{3 m+4}$ does not use arc $e_{m+3}$. Then, by properties (a) and (b), only $p_{3 m+1}, p_{3 m+2}$, and $p_{3 m+3}$ play strategies in $\left\{e_{m+1}, e_{m+2}, e_{m+3}\right\}$. However, the congestion game restricted to these three players and strategies does not have a pure-strategy Nash equilibrium, yielding a contradiction. In fact, we only need to consider all possibilities for the three players to choose their strategies from $\left\{e_{m+1}, e_{m+2}, e_{m+3}\right\}$. We can exclude from the start all possibilities that imply a cost of $K$ for one of the players. Eight possible combinations, which are listed in Table 2, remain to be considered. In consequence, the only way for the whole game to have a pure-strategy Nash equilibrium is if player $p_{3 m+4}$ uses arc $e_{m+3}$.

Given a pure-strategy Nash equilibrium $s$ of the constructed game, we can now associate a partition of the item set with the $m$ groups of players who route their flows on $\operatorname{arcs} e_{k}$ with $1 \leq k \leq m$. We claim that the so defined sets, i.e., $A_{k}:=\left\{i \in A: s_{i}=e_{k}\right\}, 1 \leq k \leq m$, form a solution to the 3-PARTITION problem. Suppose this is not true. Then there exists an index $k \in\{1,2, \ldots, m\}$ such that the total weight of players routing their flows on $e_{k}$ is at most $B-1$. Consider player $p_{3 m+4}$, who, by property (c), plays strategy $e_{m+3}$ in any pure-strategy Nash equilibrium. Her current cost is $B+1$. By switching to $\operatorname{arc} e_{k}$, she can decrease her cost to at most $B$. However, this contradicts the assumption of $s$ being a Nash equilibrium. Therefore, $\sum_{i \in A_{k}} w_{i}=B$ for $1 \leq k \leq m$.

The following result shows that deciding the existence of pure-strategy Nash equilibria in asymmetric weighted network congestion games with unsplittable flows remains strongly NP-complete, even if the number of players is fixed. Note that this result does not render Theorem 3.1 obsolete, because that theorem dealt with symmetric games.

THEOREM 3.3. The problem of deciding whether a weighted network congestion game with a fixed number of players has a pure-strategy Nash equilibrium is strongly NP-complete.

Proof. We reduce from Arc-Disjoint Paths: Given a directed graph $G=(N, A)$ and a set of node pairs $\left(r_{1}, t_{1}\right),\left(r_{2}, t_{2}\right), \ldots,\left(r_{k}, t_{k}\right)$, does there exist a collection of arc-disjoint paths $P_{1}, P_{2}, \ldots, P_{k}$, where $P_{i}$ is an $r_{i}-t_{i}$-path? This problem is NP-complete, even in the case of only two terminal pairs (Fortune et al. [15]).

Let $G=(N, A),\left(r_{1}, t_{1}\right)$, and $\left(r_{2}, t_{2}\right)$ be an instance of ArC-Disjoint Paths with two terminal pairs. We will construct a congestion game whose underlying network will consist of two building blocks. The first component is obtained from the original network $G$ by replacing each arc $a \in A$ by a path consisting of three $\operatorname{arcs} \bar{e}^{1}, \bar{e}^{2}$, and $\bar{e}^{3}$. We denote the resulting graph by $G^{\prime}$. The second building block is the network that we already used as a gadget in the proof of Theorem 3.1. It consists of four different $r$-t -paths, which we will call $Q_{k}$ here, for $k=1,2,3,4$. We will refer to it as the "lower" part of the new network. See Figure 2 for an illustration.

Both building blocks are connected as follows. Assume that $A=\left\{a_{1}, a_{2}, \ldots, a_{m}\right\}$. We introduce an arc $\bar{e}_{0}$ between $r$ and the starting node of the second arc $\bar{e}_{1}^{2}$, which was one of three arcs replacing the original arc $a_{1} \in A$. Furthermore, for $1 \leq i \leq m-1$, we create an arc $\bar{e}_{i}$ connecting the end node of $\bar{e}_{i}^{2}$ with the start node of $\bar{e}_{i+1}^{2}$. Finally, there is an arc $\bar{e}_{m}$ from the end node of $\bar{e}_{m}^{2}$ to the terminal node $t$. We denote the $r$-t $t$-path formed by the $\operatorname{arcs} \bar{e}_{k}, k=0,1, \ldots, m$, and $\bar{e}_{i}^{2}, i=1,2, \ldots, m$, by $Q_{5}$.

The game has four players. Players $p_{1}$ and $p_{2}$ wish to route one unit of flow from $r_{1}$ to $t_{1}$ and from $r_{2}$ to $t_{2}$, respectively. Players $p_{3}$ and $p_{4}$ have weights $w_{3}=m$ and $w_{4}=2 m$, to be sent from $r$ to $t$. 


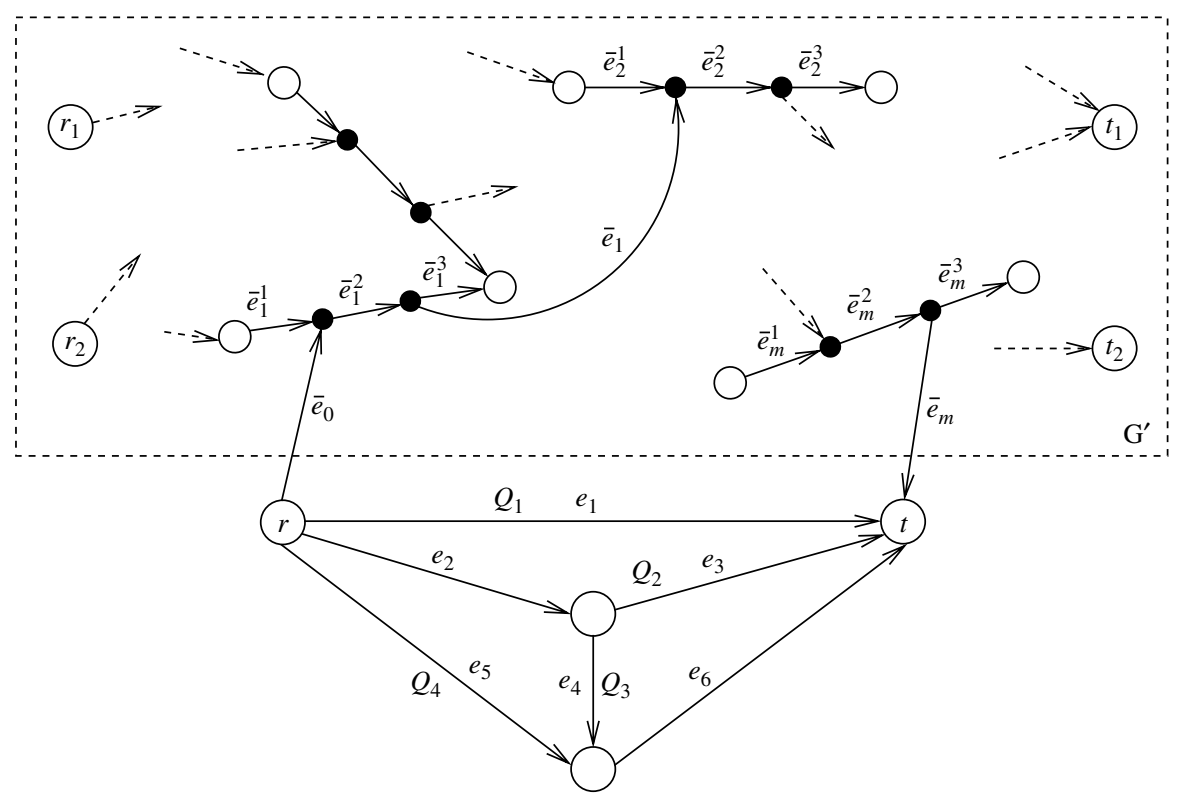

FIGURE 2. Illustration of the weighted network congestion game created in the proof of Theorem 3.3.

We define the cost of the arcs that replaced $a_{i} \in A$ as follows:

$$
\begin{aligned}
& f_{\bar{e}_{i}^{\frac{1}{i}}}(x):=f_{\bar{e}_{i}}(x):= \begin{cases}0, & \text { if } 0 \leq x<m, \\
K, & \text { if } x \geq m,\end{cases} \\
& f_{\bar{e}_{i}^{2}}(x):= \begin{cases}0, & \text { if } 0 \leq x<m+2, \\
K, & \text { if } x \geq m+2,\end{cases}
\end{aligned}
$$

where $K:=237 m+1$. Moreover, for $0 \leq i \leq m$,

$$
f_{\bar{e}_{i}}(x):= \begin{cases}0, & \text { if } 0 \leq x \leq m, \\ K, & \text { if } x>m .\end{cases}
$$

The cost functions $f_{e_{i}}$, for $i=1,2, \ldots, 6$, in the lower part of the network, are defined in the same way as the corresponding functions in the proof of Theorem 3.1; however, $\bar{B}$ is replaced by $m$.

Let us first assume that the given instance of ARC-DisJoint Paths is a Yes-instance, i.e., there exist arcdisjoint paths $P_{1}$ and $P_{2}$ in $G$ connecting $r_{1}$ and $t_{1}$, and $r_{2}$ and $t_{2}$, respectively. We will abuse notation and denote the corresponding paths in $G^{\prime}$ also by $P_{1}$ and $P_{2}$. Consider the strategy state $s$ in which players $p_{1}$ and $p_{2}$ choose $P_{1}$ and $P_{2}$, respectively, whereas player $p_{3}$ uses $Q_{5}$, and $p_{4}$ routes her flow on $Q_{2}$. We claim that $s$ is a Nash equilibrium. Because at most one of the players $p_{1}$ and $p_{2}$ uses a particular arc in $G^{\prime}$, the cost of any $\operatorname{arc} \bar{e}_{i}^{1}, \bar{e}_{i}^{2}, \bar{e}_{i}^{3}, 1 \leq i \leq m$, in $s$ is zero. Therefore, $p_{1}$ and $p_{2}$ have zero cost and play optimal strategies. Similarly, the cost of player $p_{3}$ is zero, because in addition to the last observation, the total weight on any arc $\bar{e}_{i}, 0 \leq i \leq m$, is $m$, i.e., the cost for using each of these arcs is zero. Finally, player $p_{4}$ uses the cheapest path in the lower part of the network. By routing her flow on any path sharing arcs with $G^{\prime}$, her cost would increase to at least $K$. Thus, no player can decrease her cost by routing flow over another path; $s$ is indeed a pure-strategy Nash equilibrium.

Let us now assume that we are given a pure-strategy Nash equilibrium $s$ of the constructed game. Because any arc in $G^{\prime}$, if used by player $p_{4}$, induces a cost of $K>237 \mathrm{~m}$, this player is always better off using a path in $\left\{Q_{1}, Q_{2}, Q_{3}, Q_{4}\right\}$. We further observe that there is no $r_{1}$ - $t_{1}$-path or $r_{2}$ - $t_{2}$-path that shares any arc with the lower part of the network, i.e., neither player $p_{1}$ nor player $p_{2}$ will use such an arc. However, if we restrict the game to players $p_{3}$ and $p_{4}$ and the lower part of the network, it follows from the same reasoning used in the proof of Theorem 3.1 that this subgame does not have a pure-strategy Nash equilibrium. Therefore, player $p_{3}$ has to choose a path intersecting $G^{\prime}$. Arcs $\bar{e}_{i}^{1}$ and $\bar{e}_{i}^{3}, 1 \leq i \leq m$, are very expensive if the load is greater than or equal to $w_{3}=m$; hence, the only way for $p_{3}$ to route her flow in a Nash equilibrium is to use path $Q_{5}$. Because she cannot decrease her cost by switching to a path in the lower part of the network, the cost for using 
$Q_{5}$ must be smaller than $K$. This implies that at most one of the players $p_{1}$ and $p_{2}$ uses an arc $\bar{e}_{i}^{2}, 1 \leq i \leq m$ (otherwise, the total weight on such an arc would be $m+2$ ). Similarly, neither $p_{1}$ nor $p_{2}$ can use any arc $\bar{e}_{k}$ for $k \in\{0,1, \ldots, m\}$. This, in turn, implies that both $p_{1}$ and $p_{2}$ only use $G^{\prime}$ to route their flows. Furthermore, the corresponding paths of these two players in $G$ have to be disjoint, i.e., the ARC-DisJoint Paths instance is a Yes-instance.

3.2. Integer-splittable flows. Rosenthal [32] gave an example of an asymmetric weighted network congestion game that does not have a pure-strategy Nash equilibrium if players are allowed to split their flows (see Figure 5). Interestingly, the same game possesses a pure-strategy Nash equilibrium if each player has to route her flow on a single path. The following result shows that one cannot efficiently decide the existence of pure-strategy Nash equilibria in network congestion games with integer-splittable flows, unless $\mathrm{P}=\mathrm{NP}$.

THEOREM 3.4. The problem of deciding whether a weighted network congestion game with integer-splittable flows possesses a pure-strategy Nash equilibrium is strongly NP-hard, even if there is only one player with weight two, and all other players have unit weights.

Proof. The reduction is from Monotone 3SAT, which is known to be NP-complete (Garey and Johnson [18]). Consider an instance of Monotone 3Sat with set of variables $X=\left\{x_{1}, x_{2}, \ldots, x_{n}\right\}$ and set of threevariable clauses $C=\left\{c_{1}, c_{2}, \ldots, c_{m}\right\}$. Each clause contains either only negated variables or only unnegated variables.

We will create a game that has one player $p_{x}$ for every variable $x \in X$ with weight $w_{x}=1$, origin $x$, and destination $\bar{x}$. Moreover, each clause $c \in C$ gives rise to a player $p_{c}$ with weight $w_{c}=1$, origin $c$, and destination $\bar{c}$. There are three more players $p_{1}, p_{2}$, and $p_{3}$ with weights $w_{1}=1, w_{2}=2$, and $w_{3}=1$ and origin-destination pairs $\left(r, t_{1}\right),\left(r, t_{2}\right)$, and $\left(r, t_{3}\right)$, respectively. For every variable $x \in X$, the network contains two disjoint paths $P_{x}^{1}$ and $P_{x}^{0}$ from $x$ to $\bar{x}$. Path $P_{x}^{0}$ consists of $2|\{c \in C \mid x \in c\}|+1$ arcs, and $P_{x}^{1}$ has $2|\{c \in C \mid \bar{x} \in c\}|+1$ arcs with cost functions as shown in Figure 3. For each origin-destination pair $(c, \bar{c})$, we introduce two disjoint paths $P_{c}^{1}$ and $P_{c}^{0}$ from $c$ to $\bar{c}$. Path $P_{c}^{1}$ consists of only two arcs. The paths $P_{c}^{0}$ have seven arcs each and are constructed for $c=c_{j}$ in the order $j=1,2, \ldots, m$ as follows. For a positive clause $c=c_{j}=\left(x_{j_{1}} \vee x_{j_{2}} \vee x_{j_{3}}\right)$ with $j_{1}<j_{2}<j_{3}$, path $P_{c}^{0}$ starts with the arc connecting $c$ to the first inner node $v_{1}$ on path $P_{x_{j_{1}}}^{1}$ that has only two incident arcs so far. The second arc is the unique arc $\left(v_{1}, v_{2}\right)$ of path $P_{x_{j_{1}}}^{1}$ that has $v_{1}$ as its start vertex. The third arc connects $v_{2}$ to the first inner node $v_{3}$ on path $P_{x_{j_{2}}}^{1}$ that has only two incident arcs so far. The fourth arc is the only arc $\left(v_{3}, v_{4}\right)$ on $P_{x_{j 2}}^{1}$ with start vertex $v_{3}$. From $v_{4}$, there is an arc to the first inner node $v_{5}$ on $P_{x_{j_{3}}}^{1}$ that has only two incident arcs so far, followed by $\left(v_{5}, v_{6}\right)$ of $P_{x_{j_{3}}}^{1}$. The last arc of $P_{c}^{0}$ connects $v_{6}$ to $\bar{c}$. Figure 3 illustrates this construction. For a negative clause $c=c_{j}=\left(\bar{x}_{j_{1}} \vee \bar{x}_{j_{2}} \vee \bar{x}_{j_{3}}\right)$, we proceed in the same way, except that we choose the inner nodes $v_{i}$ from the upper variable paths $P_{x_{j_{1}}}^{0_{2}}, P_{x_{j_{2}}}^{0}$, and $P_{x_{j_{3}}}^{0}$.

The strategy set of player $p_{x}$ is $\left\{P_{x}^{1}, P_{x}^{0}\right\}$. We will interpret it as setting the variable $x$ to true (false) if $p_{x}$ sends her unit of flow over $P_{x}^{1}\left(P_{x}^{0}\right)$. Note that player $p_{c}$ can only choose between the paths $P_{c}^{1}$ and $P_{c}^{0}$. This is due to the order in which the paths $P_{c}^{0}$ are constructed. Depending on whether player $p_{c}$ sends her unit of flow over path $P_{c}^{1}$ or $P_{c}^{0}$, the clause $c$ will be satisfied or not.

The second part of the network consists of all origin-destination pairs and paths for players $p_{1}, p_{2}$, and $p_{3}$ (see Figure 4). Player $p_{1}$ can choose between paths $U_{1}=\left\{\left(r, t_{2}\right),\left(t_{2}, t_{1}\right)\right\}$ and $L_{1}=\left\{\left(r, t_{1}\right)\right\}$. Player $p_{2}$ is the only

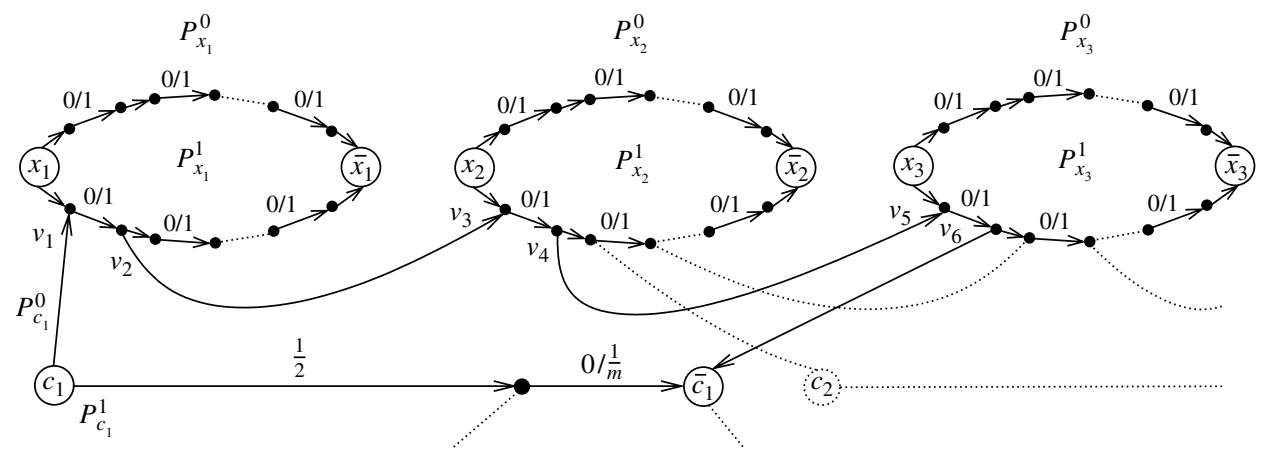

FIgURE 3. Part of the constructed network corresponding to a positive clause $c_{1}=\left(x_{1} \vee x_{2} \vee x_{3}\right)$. The notation $a / b$ defines a cost per unit flow of value $a$ for load 1 and $b$ for load 2. For any other arc, the cost does not depend on the amount of flow on that arc. Arcs without specified values have zero cost. 


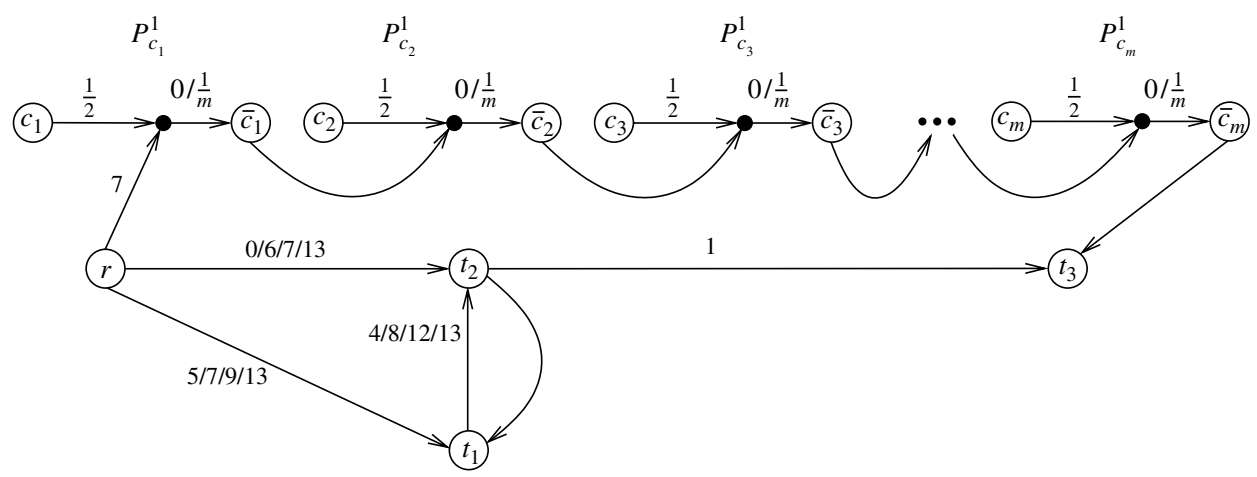

Figure 4. Part of the network used by players $p_{1}, p_{2}$, and $p_{3}$. A single number $a$ on an arc defines a constant cost of $a$ per unit flow for this arc. Unlabeled arcs have cost zero.

player who can split her flow; that is, she can route her two units either both over path $U_{2}=\left\{\left(r, t_{2}\right)\right\}$, both over path $L_{2}=\left\{\left(r, t_{1}\right),\left(t_{1}, t_{2}\right)\right\}$, or one unit on the upper and the other unit on the lower path; i.e., her strategy set is $S_{2}=\left\{L_{2}, U_{2}, L_{2} U_{2}\right\}$. Finally, player $p_{3}$ has three possible paths from which to choose. The upper path $U_{3}$ shares an arc with each clause path $P_{c}^{1}$ and has some additional arcs to connect these. The paths $M_{3}=\left\{\left(r, t_{2}\right),\left(t_{2}, t_{3}\right)\right\}$ and $L_{3}=\left\{\left(r, t_{1}\right),\left(t_{1}, t_{2}\right),\left(t_{2}, t_{3}\right)\right\}$ have only arcs with the paths of $p_{1}$ and $p_{2}$ in common. The cost functions are defined in Figure 4.

Given a satisfying truth assignment, we define a strategy state $s$ of the game by setting the strategy of player $p_{x}$ to be $P_{x}^{1}$ for a true variable $x$, and $P_{x}^{0}$ otherwise. Each player $p_{c}$ plays $P_{c}^{1}$. Furthermore, $s_{1}=L_{1}, s_{2}=U_{2}$, and $s_{3}=M_{3}$. It is easy to show that no player can decrease her cost by unilaterally switching to another strategy; i.e., the defined strategy configuration is a pure-strategy Nash equilibrium.

For the other direction, we first observe that any pure-strategy Nash equilibrium $s$ has the following properties: (a) player $p_{3}$ does not use path $U_{3}$, (b) the cost of player $p_{3}$ is at least eight, and (c) each player $p_{c}$ routes her unit flow over path $P_{c}^{1}$. Property (a) follows from the fact that the subgame shown in Figure 5 with players $p_{1}$ and $p_{2}$ only does not have a pure-strategy Nash equilibrium. ${ }^{4}$ Thus, $p_{3}$ will use either the middle or the lower path. No matter how many other players use an edge of the lower path, the cost of $p_{3}$ using $L_{3}$ is at least 10 . The only possibility for $p_{3}$ to face a cost strictly less than eight is if she uses the middle path and at most one additional unit of $p_{1}$ or $p_{2}$ is routed over arc $\left(r, t_{2}\right)$. Let us consider the case $s_{1}=L_{1}, s_{2}=L_{2}$, and $s_{3}=M_{3}$ first. Then $p_{2}$ has a cost of 34, and she can decrease her cost by switching to strategy $U_{2}$ with a new cost of 14 . If $s_{1}=L_{1}, s_{2}=L_{2} U_{2}$, and $s_{3}=M_{3}$, the cost of player $p_{2}$ is 17 . Choosing strategy $U_{2}$ instead yields a lower cost of 14. The last case to consider is $s_{1}=U_{1}, s_{2}=L_{2}$, and $s_{3}=M_{3}$. Then, player $p_{2}$ has a cost of 30, which can be decreased by switching to strategy $L_{2} U_{2}$, leading to a cost of 16 . Consequently, property (b) holds for any pure-strategy Nash equilibrium of the game. For property (c), suppose there is a player $p_{c}, c \in C$, routing her unit flow on $P_{c}^{0}$. By (a) and (b), we know that $p_{3}$ uses either the lower or the middle path with a cost of at least eight. Consider a change of $p_{3}$ to the upper path $U_{3}$. Her new cost would be at most $7+(m-1)(1 / m)<8$, which would contradict that $s$ is a Nash equilibrium.

We claim that the truth assignment that sets a variable $x$ to true if the corresponding player uses $P_{x}^{1}$, and $x$ to false otherwise, satisfies all clauses. Suppose that all variables of a positive clause $c=\left(x_{1} \vee x_{2} \vee x_{3}\right)$ are false; i.e., $s_{x_{i}}=P_{x_{i}}^{0}$ for $i=1,2,3$. By property (c), player $p_{c}$ uses $P_{c}^{1}$. Because of (a), her current cost is $1 / 2$. Choosing path $P_{c}^{0}$ instead would decrease the cost to zero, which contradicts the assumption of $s$ being a Nash equilibrium. A similar argument holds for a negative clause.

Note that we have not claimed that the problem of deciding whether a weighted network congestion game with integer-splittable flows possesses a pure-strategy Nash equilibrium is in NP. Although this can be easily shown if all cost functions are convex, it follows from a result by Meyers and Schulz [26] that, in general, the problem of deciding whether a given strategy profile is a pure-strategy Nash equilibrium is in itself a co-NP-complete problem.

4. Bidirectional local-effect games. Leyton-Brown and Tennenholtz [24] presented a characterization of local-effect games that have an exact potential function and that are therefore guaranteed to possess purestrategy Nash equilibria. One of these subclasses is that of bidirectional local-effect games with linear local-effect

${ }^{4}$ This subgame coincides with the instance originally conceived by Rosenthal [32], to which we alluded earlier. 

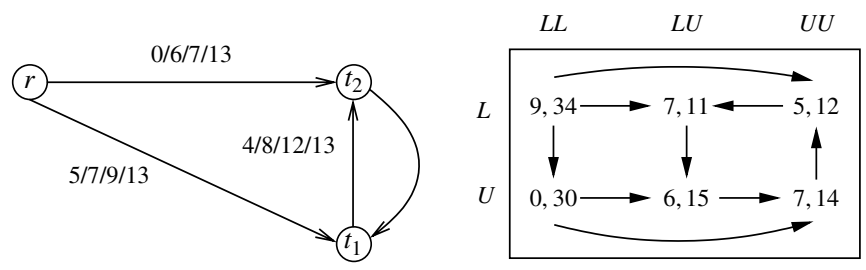

Figure 5. On the left: Subgame with two players without pure-strategy Nash equilibrium. On the right: Digraph of all strategy states and improving moves.

Note. The players' weights are one and two, respectively.

functions. However, without linear local-effect functions, deciding the existence of pure-strategy Nash equilibria is difficult.

THEOREM 4.1. The problem of deciding whether a bidirectional local-effect game possesses a pure-strategy Nash equilibrium is strongly NP-complete.

Proof. The proof uses a reduction from 3-PARTITION. Consider an arbitrary instance of 3-PARTition with finite set $A=\{1,2, \ldots, 3 m\}$ of items, a number $B \in \mathbb{N}$, and a positive integer weight $w_{i}$ for each item $i \in A$ such that $B / 4<w_{i}<B / 2$ and $\sum_{i \in A} w_{i}=m B$. We may assume that $B \geq 12$. We will construct a bidirectional local-effect game such that it has a pure-strategy Nash equilibrium if and only if $A$ can be partitioned into $m$ disjoint sets $A_{1}, A_{2}, \ldots, A_{m}$ such that $\sum_{i \in A_{k}} w_{i}=B$ for $1 \leq k \leq m$.

The action set $\mathscr{A}$ consists of $3 m^{2}+2 m+3$ actions that are available to $4 m+3$ players. For each item $i \in A$, there is a set of $m$ corresponding actions $a_{i}^{1}, a_{i}^{2}, \ldots, a_{i}^{m}$; we will make sure that in any Nash equilibrium of the game exactly one player will pick one of these actions, for each item. The remaining actions are denoted by $d_{j}$ and $\bar{d}_{j}, j=1,2, \ldots, m$, and $h_{1}, h_{2}$, and $h_{3}$. We define the cost functions for actions $a \in\left\{a_{i}^{j}: i=\right.$ $1,2, \ldots, 3 m, j=1,2, \ldots, m\} \cup\left\{d_{j}: j=1,2, \ldots, m\right\}$ as

$$
f_{a}(x):= \begin{cases}0, & \text { if } x \leq 1, \\ K, & \text { otherwise }\end{cases}
$$

where $K:=3(4 m+3) B+18$. For $j=1,2, \ldots, m$,

$$
f_{\bar{d}_{j}}(x):= \begin{cases}B, & \text { if } x \leq 1, \\ K, & \text { otherwise. }\end{cases}
$$

Furthermore, we have

$$
\begin{aligned}
f_{h_{1}}(x):=f_{h_{2}}(x):= \begin{cases}0, & \text { if } x=0, \\
B+1, & \text { if } x=1, \\
2 B+6, & \text { if } x=2, \\
3 B+12, & \text { if } x=3, \\
B x, & \text { otherwise, }\end{cases} \\
f_{h_{3}}(x):= \begin{cases}0, & \text { if } x=0, \\
B+1, & \text { if } x=1, \\
2 B+2, & \text { if } x=2, \\
3 B+12, & \text { if } x=3, \\
B x, & \text { otherwise. }\end{cases}
\end{aligned}
$$

The local-effect functions between actions $h_{1}, h_{2}$, and $h_{3}$ are given by

$$
f_{h_{1}, h_{2}}(x):= \begin{cases}0, & \text { if } x=0, \\ B+1, & \text { if } x=1, \\ 2 B+4, & \text { if } x=2, \\ B x, & \text { otherwise, }\end{cases}
$$




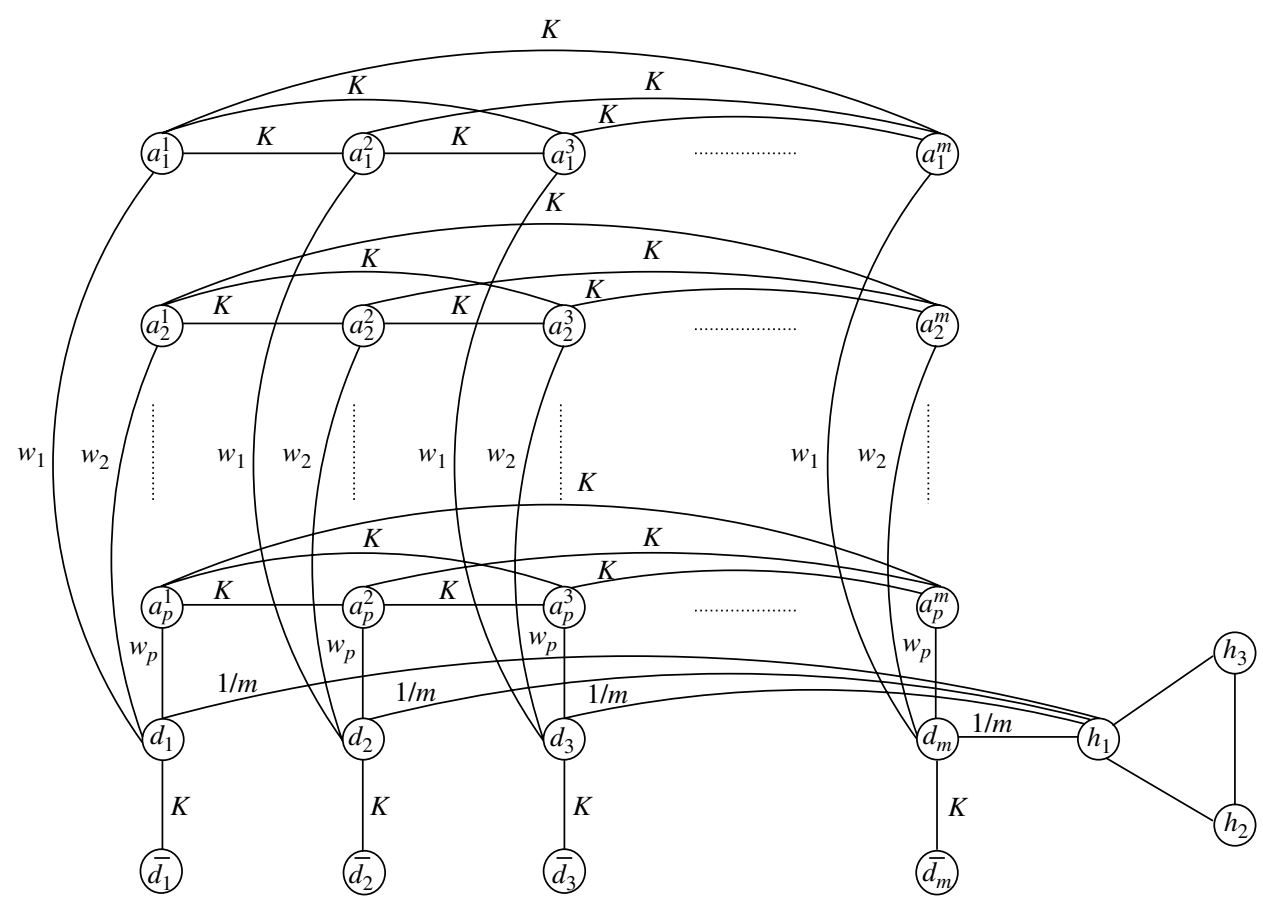

FIGURE 6. Illustration of the "local-effect graph" of the game constructed in the proof of Theorem 4.1. Note. An arc label $\alpha$ corresponds to the coefficient of a linear local-effect function $f_{a, a^{\prime}}(x)=\alpha x$. Moreover, $p:=3 m$.

$$
\begin{aligned}
& f_{h_{1}, h_{3}}(x):= \begin{cases}0, & \text { if } x=0, \\
B+3, & \text { if } x=1, \\
2 B+4, & \text { if } x=2, \\
B x, & \text { otherwise, }\end{cases} \\
& f_{h_{2}, h_{3}}(x):= \begin{cases}0, & \text { if } x=0, \\
B+1, & \text { if } x=1, \\
2 B+5, & \text { if } x=2, \\
B x, & \text { otherwise. }\end{cases}
\end{aligned}
$$

All other local-effect functions are defined in Figure 6.

Assume that the given 3-PARTITION instance is a Yes-instance; that is, there is a partition $A_{1}, A_{2}, \ldots, A_{m}$ of the item set $A$ such that $\sum_{i \in A_{k}} w_{i}=B$ for $1 \leq k \leq m$. Consider the following strategy state $s$ of the local-effect game. For $i=1,2, \ldots, 3 m$ and $j=1,2, \ldots, m$, let

$$
n_{d_{j}}(s)=1, \quad n_{\bar{d}_{j}}(s)=0, \quad n_{h_{1}}(s)=0, \quad n_{h_{2}}(s)=1, \quad n_{h_{3}}(s)=2, \quad \text { and } \quad n_{a_{i}^{j}}(s)= \begin{cases}1, & \text { if } i \in A_{j}, \\ 0, & \text { otherwise } .\end{cases}
$$

We show that no player can decrease her cost by switching to another action. First, a player choosing some action $a_{i}^{j}$ pays a cost of $w_{i}$ due to a local effect from action $d_{j}$. Switching to action $a_{i}^{k}$ for some $k \neq j$ does not change the player's cost. Any other action apart from $h_{1}, h_{2}$, or $h_{3}$ would imply a cost of at least $K$. Switching to action $h_{1}, h_{2}$, or $h_{3}$ leads to a cost of at least $B+1>w_{i}$. Thus, none of these players can decrease her cost. Now consider a player with action $d_{j}$. Her cost is $\sum_{i \in A_{j}} w_{i}=B$. Switching to some action $a_{i}^{j}, d_{k}$, or $\bar{d}_{k}$ with $k \neq j$ increases her cost to at least $K$. Action $\bar{d}_{j}$ implies an equal cost of $B$. Switching to $h_{1}, h_{2}$, or $h_{3}$ results in a cost of at least $B+1$. Hence, such a player has no incentive to change her strategy either. The player playing action $h_{2}$ has a cost of $3 B+6$. Switching to some action in $\mathscr{A} \backslash\left\{h_{1}, h_{2}, h_{3}\right\}$ increases her cost to at least $K$. A change to action $h_{1}$ or $h_{3}$ results in a new cost of $3 B+6$ or $3 B+12$, respectively. Finally, a player with action $h_{3}$ has cost $3 B+3$ in state $s$. She would also strictly increase her cost by switching to some action in $\mathscr{A} \backslash\left\{h_{1}, h_{2}, h_{3}\right\}$. A change to action $h_{1}$ would result in a cost of $3 B+6$, whereas switching to $h_{2}$ increases her cost by four cost units. Consequently, the defined state is a pure-strategy Nash equilibrium. 
TABLE 3. Possible defections in the subgame restricted to actions $h_{1}, h_{2}$, and $h_{3}$ and three players “..., under the assumption that $\sum_{j=1}^{m} n_{d_{j}}(s)<m$ "

\begin{tabular}{lccccc}
\hline$n_{h_{1}}$ & $n_{h_{2}}$ & $n_{h_{3}}$ & Defector $\rightarrow$ New strategy & Current cost & Improved cost \\
\hline 3 & 0 & 0 & $h_{1} \rightarrow h_{2}$ & $\geq 3 B+12$ & $3 B+5$ \\
0 & 3 & 0 & $h_{2} \rightarrow h_{3}$ & $3 B+12$ & $3 B+6$ \\
0 & 0 & 3 & $h_{3} \rightarrow h_{2}$ & $3 B+12$ & $3 B+6$ \\
2 & 1 & 0 & $h_{1} \rightarrow h_{3}$ & $\geq 3 B+7$ & $3 B+5$ \\
2 & 0 & 1 & $h_{1} \rightarrow h_{2}$ & $\geq 3 B+9$ & $3 B+3$ \\
1 & 2 & 0 & $h_{2} \rightarrow h_{3}$ & $3 B+7$ & $3 B+5$ \\
0 & 2 & 1 & $h_{2} \rightarrow h_{3}$ & $3 B+7$ & $3 B+3$ \\
1 & 0 & 2 & $h_{3} \rightarrow h_{2}$ & $3 B+5$ & $3 B+3$ \\
0 & 1 & 2 & $h_{2} \rightarrow h_{1}$ & $3 B+6$ & $\leq 3 B+5+(m-1) / m$ \\
1 & 1 & 1 & $h_{1} \rightarrow h_{3}$ & $\geq 3 B+5$ & $3 B+3$ \\
\hline
\end{tabular}

For the other direction of the proof, we make the following observations. Let $s$ be a pure-strategy Nash equilibrium. Then, for each $i \in\{1,2, \ldots, 3 m\}$, at most one player chooses a strategy from the set $\left\{a_{i}^{j}: j \in\right.$ $\{1,2, \ldots, m\}\}$. Otherwise, at least one of the players could decrease her current cost of at least $K$ to less than $3(4 m+3) B<K$ by switching to action $h_{3}$. By the same token, for each $j \in\{1,2, \ldots, m\}$, at most one player chooses an action from $\left\{d_{j}, \bar{d}_{j}\right\}$ in $s$. It follows that at least three players play an action from $\left\{h_{1}, h_{2}, h_{3}\right\}$. Suppose there were more than three. Then, there existed either an $i \in\{1,2, \ldots, 3 m\}$ such that $\sum_{j=1}^{m} n_{a_{i}^{j}}(s)=0$ or some $j \in\{1,2, \ldots, m\}$ with $n_{d_{j}}(s)+n_{\bar{d}_{j}}(s)=0$. In the first case, one of the players currently playing $h_{1}$, $h_{2}$, or $h_{3}$ could decrease her cost from at least $B+1$ to at most $w_{i}<B$ if she made the switch to any of the actions corresponding to item $a_{i}$. In the second case, switching to $\bar{d}_{j}$ would decrease the cost of some player to $B$. Consequently,

$$
\begin{gathered}
n_{h_{1}}(s)+n_{h_{2}}(s)+n_{h_{3}}(s)=3, \\
\sum_{j=1}^{m} n_{a_{i}^{j}}(s)=1 \quad \text { for } i=1,2, \ldots, 3 m, \quad \text { and } \\
n_{d_{j}}(s)+n_{\bar{d}_{j}}(s)=1 \quad \text { for } j=1,2, \ldots, m .
\end{gathered}
$$

The key insight left to show is that $n_{d_{j}}(s)=1$ for $j=1,2, \ldots, m$. Assume that $\sum_{j=1}^{m} n_{d_{j}}(s)<m$. Consider the subgame defined by actions $h_{1}, h_{2}$, and $h_{3}$ and three players. Table 3 shows that this subgame does not have a pure-strategy Nash equilibrium. Therefore, the only way $s$ can be a pure-strategy Nash equilibrium is that the local effects on $h_{1}$ due to actions $d_{j}$ sum up to one (see the row next to last in Table 3). Consequently, $n_{d_{j}}(s)=1$ for $j=1,2, \ldots, m$.

Now consider a player with action $d_{j}$ in state $s$. Because $s$ is a Nash equilibrium, she cannot decrease her cost by switching to action $\bar{d}_{j}$. Therefore, the current cost of this player has to satisfy $\sum_{i=1}^{3 m} n_{a_{i}^{j}}(s) w_{i} \leq B$ (note that by (3), $\left.n_{\bar{d}_{j}}(s)=0\right)$. Because of (2), we have $\sum_{i=1}^{3 m} \sum_{j=1}^{m} n_{a_{i}^{j}}(s) w_{i}=m B$, implying

$$
\sum_{i=1}^{3 m} n_{a_{i}^{j}}(s) w_{i}=B \quad \text { for } j=1,2, \ldots, m
$$

Hence, a solution to the partitioning problem is given by $A_{j}:=\left\{i \in A: n_{a_{i}^{j}}(s)=1\right\}, j=1,2, \ldots, m$.

If the number of players or the number of actions in a local-effect game is fixed, then the number of possible strategy combinations of all players is polynomially bounded in the input size. Hence, deciding the existence of pure-strategy Nash equilibria is solvable in polynomial time in these cases.

As mentioned before, bidirectional local-effect games with linear local-effect functions belong to the class of exact potential games (Leyton-Brown and Tennenholtz [24]); in particular, pure-strategy Nash equilibria are guaranteed to exist. However, it turns out that computing one is at least as hard as finding a local optimum for several combinatorial optimization problems with efficiently searchable neighborhoods.

THEOREM 4.2. The problem of computing pure-strategy Nash equilibria for bidirectional local-effect games with linear local-effect functions is PLS-complete. 
Proof. We reduce from Max-Cut with the flip-neighborhood, which is PLS-complete (Schäffer and Yannakakis [33]): ${ }^{5}$ Given a complete graph on $n$ nodes with nonnegative integral edge weights $w_{i j}$, for $i \neq j$, find a partition of the nodes into two sets $L$ and $R$ such that the sum of the weights of edges between nodes in $L$ and $R$ cannot be increased by moving a single node from one set to the other.

We construct a bidirectional local-effect game with linear local-effect functions as follows. There are $n$ players with common action set $\mathscr{A}$ that contains two actions $a_{i}^{L}$ and $a_{i}^{R}$ for each node $i=1,2, \ldots, n$. Let $K:=n \max _{i \neq j} w_{i j}$. For each action $a \in \mathscr{A}, f_{a}(x):=0$ if $x \leq 1$, and $f_{a}(x):=K$, otherwise. The local-effect functions are given for $i, j \in\{1,2, \ldots, n\}, i \neq j$, by $f_{a_{i}^{L}, a_{j}^{L}}(x):=f_{a_{i}^{R}, a_{j}^{R}}(x):=w_{i j} x$. Furthermore, $f_{a_{i}^{L}, a_{i}^{R}}(x):=K x$ for $i \in\{1,2, \ldots, n\}$. All local-effect functions not defined so far are identical to zero. This definition of local effects ensures that in any pure-strategy Nash equilibrium $s$ of the game,

$$
n_{a_{i}^{L}}(s)+n_{a_{i}^{R}}(s)=1 \quad \text { for } i=1,2, \ldots, n .
$$

For if not, at least one player would be able to decrease her present cost of at least $K$ by switching from her current action to an action $a_{i}^{L}$ for which $n_{a_{i}^{L}}(s)+n_{a_{i}^{R}}(s)=0$. Ergo, we can associate with any pure-strategy Nash equilibrium $s$ a unique cut in the graph by defining $L(s):=\left\{i: n_{a_{i}^{L}}(s)=1\right\}$ and $R(s):=\left\{i: n_{a_{i}^{R}}(s)=1\right\}$.

We show next that for any pure-strategy Nash equilibrium $s$ of the game, the corresponding cut is indeed a local optimum of the MAX-CUT instance. Because $s$ is a Nash equilibrium, no player can decrease her cost by switching to another action. In particular, a player with action $a_{i}^{L}, i \in\{1,2, \ldots, n\}$, cannot improve by switching to action $a_{i}^{R}$. Using (4), this implies

$$
\sum_{\substack{j=1 \\ j \neq i}}^{n} w_{i j} n_{a_{j}^{L}}(s) \leq \sum_{\substack{j=1 \\ j \neq i}}^{n} w_{i j} n_{a_{j}^{R}}(s) .
$$

With the definition of $L(s)$ and $R(s)$, it follows that $\sum_{j \in R(s)} w_{i j}-\sum_{j \in L(s)} w_{i j} \geq 0$. Thus, moving node $i$ from $L(s)$ to $R(s)$ does not increase the weight of the associated cut. Similarly, one can show that shifting a node from $R(s)$ to $L(s)$ cannot improve the cut either. We may conclude that the described transformation is indeed a PLS-reduction.

Because the reduction is actually a tight PLS-reduction, we obtain the following two results for free (see, e.g., Yannakakis [36]).

COROLlaRY 4.1. There are instances of bidirectional local-effect games with linear local-effect functions that have exponentially long shortest improvement paths.

COROLlary 4.2. For a bidirectional local-effect game with linear local-effect functions, the problem of finding a pure-strategy Nash equilibrium that is reachable from a given strategy state via selfish improvement steps is PSPACE-complete.

The following observation underlines that finding a pure-strategy Nash equilibrium for bidirectional localeffect games with linear local-effect functions is indeed hard. It was inspired by similar results of Fischer [14] for some local search problems.

THeOREM 4.3. Given an instance of a bidirectional local-effect game with linear local-effect functions, a pure-strategy profile $s_{0}$, and an integer $k>0$ (unarily encoded), it is strongly NP-complete to decide whether there exists a sequence of at most $k$ selfish steps that transforms $s_{0}$ into a pure-strategy Nash equilibrium.

Proof. Given a sequence $\left(s_{0}, s_{1}, \ldots, s_{n}\right)$ of pure-strategy profiles, we can check in polynomial time whether it is a sequence of at most $k$ self-improving steps such that $s_{n}$ is a pure-strategy Nash equilibrium, i.e., the problem is in NP. The proof of strong NP-completeness is by reduction from 3-PARTITION.

Consider an arbitrary instance of 3-PARTITION with item set $A=\{1,2, \ldots, 3 m\}$, positive integer weights $w_{i}$ with $B / 4<w_{i}<B / 2$ for $i \in A$, where $B \in \mathbb{N}$ and $\sum_{i \in A} w_{i}=m B$. Without loss of generality, we may assume that $m>2$. We construct an instance of a bidirectional local-effect game with linear local-effect functions and

\footnotetext{
${ }^{5}$ Our original proof of this result (Dunkel and Schulz [11]) used a reduction from PosNAE3FuIP (Schäffer and Yannakakis [33]). The new, shorter proof presented here was inspired by a proof of Ackermann et al. [1] for the PLS-completeness of finding a pure-strategy Nash equilibrium in congestion games with linear cost functions. In fact, it was pointed out by an anonymous referee that there is a close relationship between congestion games with linear cost functions and local-effect games with linear local-effect functions. In particular, the following reduction shows that the result by Ackermann et al. [1] is stronger than Theorem 4.2. Given a congestion game with strategy sets $S_{1}, S_{2}, \ldots, S_{n} \subseteq 2^{E}$, let $m_{e}$ be the slope of the linear cost function associated with resource $e \in E$. Define a bidirectional local-effect game as follows: The action set is $\mathscr{A}:=\bigcup_{i=1}^{n} S_{i}$. Moreover, for $a \in \mathscr{A}, f_{a}(x):=\sum_{e \in a} m_{e} x+M(x-1)$. In addition, $f_{a^{\prime}, a}(x):=\sum_{e \in a \cap a^{\prime}} m_{e} x$ if $a \in S_{i}$ and $a^{\prime} \in S_{j}$ with $i \neq j$. If $a, a^{\prime} \in S_{i}$, then $f_{a^{\prime}, a}(x):=M x$. Here, $M$ is a sufficiently large constant.
} 
a pure-strategy profile $s_{0}$ such that there exists a sequence of at most $k=4(m-1)$ selfish steps that transforms $s_{0}$ into a pure-strategy Nash equilibrium if and only if the 3-PARTITION instance is a Yes-instance, i.e., $A$ can be partitioned into $m$ disjoint subsets $A_{1}, A_{2}, \ldots, A_{m}$ such that $\sum_{i \in A_{j}} w_{i}=B$ for $j=1,2, \ldots, m$.

The action set $\mathscr{A}$ of the local-effect game contains for each item $i \in A$ actions $a_{i}^{j}$ for $j=1,2, \ldots, m$. In addition, there are actions $a^{j}$ for $j=1,2, \ldots, m$, and actions $b_{l}$ for $l=1,2, \ldots, k+1$. To simplify notation, we introduce index sets $J:=\{1,2, \ldots, m\}, L_{1}:=\{1,2, \ldots, m-1\}, L_{2}:=\{m, m+1, \ldots, k+1\}$, and $L:=L_{1} \cup L_{2}$. The game has $3 m+k+2$ players. We also let $D:=k B+1$ and $K:=D^{2}+1$. Then, the cost functions are defined as follows:

$$
\begin{aligned}
& f_{a^{j}}(x):=D+\frac{x-1}{k+2} \\
& f_{a_{i}^{j}}(x):=\left\{\begin{array}{ll}
x, & \text { if } x \leq 1, \\
K x, & \text { otherwise, }
\end{array} \text { for all } i \in A \text { and } j \in J,\right. \\
& f_{b_{l}}(x):=\left\{\begin{array}{ll}
(D+B+1) x, & \text { if } x \leq 1, \\
K x, & \text { otherwise, }
\end{array} \text { for all } l \in L_{1},\right. \\
& f_{b_{l}}(x):= \begin{cases}(D+B) x, & \text { if } x \leq 1, \\
K x, & \text { otherwise. }\end{cases}
\end{aligned}
$$

For the slopes of the linear local-effect functions, the reader is referred to Figure 7 . Finally, we define the pure-strategy profile $s_{0}$ by

$$
\begin{array}{ll}
n_{a^{1}}\left(s_{0}\right):=1, & \\
n_{a^{j}}\left(s_{0}\right):=0 & \text { for all } j \in J \backslash\{1\}, \\
n_{a_{i}^{1}}\left(s_{0}\right):=1 & \text { for all } i \in A, \\
n_{a_{i}^{j}}\left(s_{0}\right):=0 & \text { for all } i \in A \text { and } j \in J \backslash\{1\}, \\
n_{b_{l}}\left(s_{0}\right):=1 & \text { for all } l \in L .
\end{array}
$$

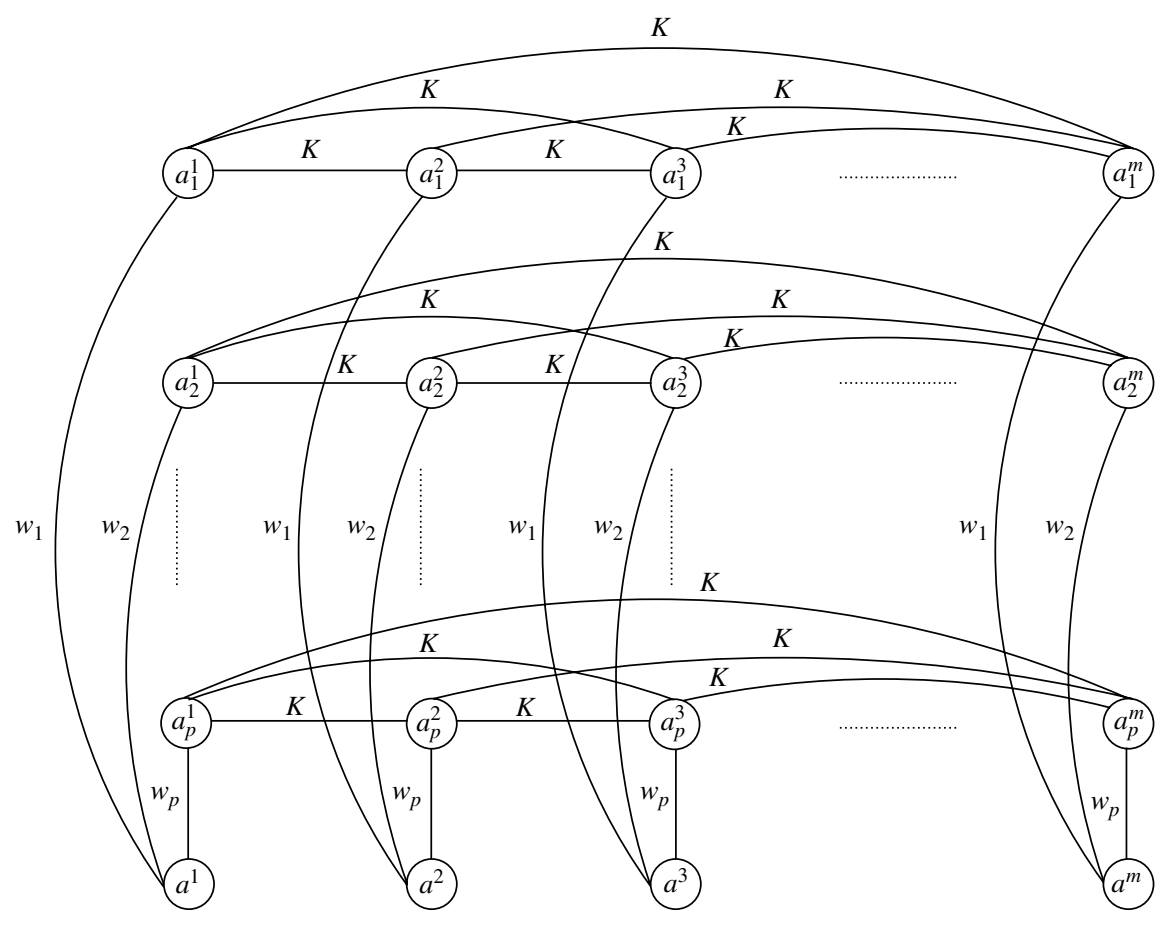


Assume that the 3-PARTITION instance is a Yes-instance. We consider the following sequence of players' moves. First, the $3(m-1)$ players with an action $a_{i}^{1}$ in $s_{0}$ such that $i \in A_{j}$ for some $j \neq 1$ switch to action $a_{i}^{j}$, in arbitrary order. They are followed by the $m-1$ players with actions $b_{l}, l \in L_{1}$, who move, in arbitrary order, to actions $a^{l+1}$. It is not difficult to see that this sequence has the desired properties. Furthermore, the resulting state $s$, i.e.,

$$
\begin{aligned}
& n_{a^{j}}(s)=1 \quad \text { for all } j \in J, \\
& n_{a_{i}^{j}}(s)=\left\{\begin{array}{ll}
1, & \text { if } i \in A_{j}, \\
0, & \text { otherwise, }
\end{array} \quad \text { for all } i \in A \text { and } j \in J,\right. \\
& n_{b_{l}}(s)=0 \quad \text { for all } l \in L_{1}, \\
& n_{b_{l}}(s)=1 \quad \text { for all } l \in L_{2} \text {. }
\end{aligned}
$$

defines a pure-strategy Nash equilibrium of the game.

For the other direction, assume that there exists a sequence of at most $k$ self-improving steps that transforms $s_{0}$ into a pure-strategy Nash equilibrium $s$ of the local-effect game. We claim that $s$ satisfies

$$
\sum_{j \in J} n_{a_{i}^{j}}(s)=1 \quad \text { for all } i \in A .
$$

It cannot happen that $\sum_{j \in J} n_{a_{i}^{j}}(s)>1$ for some $i \in A$ because then there would be a player with cost at least $K$, who could certainly improve. Therefore, suppose there is an $i \in A$ such that $\sum_{j \in J} n_{a_{i}^{j}}(s)=0$. Then, at least $k+3$ players play an action in $\left\{a^{j} \mid j \in J\right\} \cup\left\{b_{l} \mid l \in L\right\}$, each having a cost of at least $D$. Because there is some $j \in J$ such that $n_{a^{j}}(s) \leq(3 m+k+2) / m \leq 7$, any one of these players can decrease her cost by switching to action $a_{i}^{j}$ with new cost at most $1+7 w_{i}<D$. This is a contradiction, and hence (6) must hold. Consequently,

$$
\sum_{i \in A} w_{i}\left(\sum_{j \in J} n_{a_{i}^{j}}(s)\right)=m B
$$

In the last step of the proof, we show that

$$
\sum_{i \in A} w_{i} n_{a_{i}^{j}}(s)=B \quad \text { for all } j \in J
$$

i.e., $A_{j}:=\left\{i \in A \mid n_{a_{i}^{j}}(s)=1\right\}$ defines a solution to the partition problem. Suppose (7) does not hold. Then there is some $j \in J$ with

$$
\sum_{i \in A} w_{i} n_{a_{i}^{j}}(s) \leq B-1 .
$$

We claim that this implies $n_{b_{l}}(s)=0$ for all $l \in L$. If there was an $l$ with $n_{b_{l}}(s)=1$, then the cost of the corresponding player would be at least $D+B$. Because $s$ was obtained from $s_{0}$ by a sequence of at most $k$ steps, and because $n_{a^{j}}\left(s_{0}\right) \leq 1$ for all $j \in J$, we have $n_{a^{j}}(s) \leq k+1$. The player with action $b_{l}$ can decrease her cost by changing to action $a^{j}$, because $f_{a^{j}}(k+2)+\sum_{i \in A} w_{i} n_{a_{i}^{j}}(s)<D+B$. Therefore, $n_{b_{l}}(s)=0$ for all $l \in L$. However, because of $n_{b_{l}}\left(s_{0}\right)=1$ for all $l \in L$, at least $k+1$ players have to change their action in order to reach strategy state $s$. This is a contradiction; i.e., (7) must hold, and the sets $A_{j}$ as defined above are a feasible solution to the 3-PARTition instance.

Acknowledgments. The authors thank Tim Roughgarden for bringing an article by Rosenthal [32] to their attention. They also thank two anonymous referees for their helpful comments. This work was supported by National Science Foundation (NSF) Grant 0426686 and Office of Naval Research (ONR) Grant N00014-05-1-0165.

\section{References}

[1] Ackermann, H., H. Röglin, B. Vöcking. 2006a. On the impact of combinatorial structure on congestion games. Proc. 47th Ann. IEEE Sympos. Foundations Comput. Sci., Berkeley, CA, 613-622.

[2] Ackermann, H., H. Röglin, B. Vöcking. 2006b. Pure Nash equilibria in player-specific and weighted congestion games. P. G. Spirakis, M. Mavronicolas, S. C. Kontogiannis, eds. Proc. 2nd Internat. Workshop on Internet and Network Econom. Lecture Notes in Computer Science, Vol. 4286. Springer, Berlin, 50-61. 
[3] Aland, S., D. Dumrauf, M. Gairing, B. Monien, F. Schoppmann. 2006. Exact price of anarchy for polynomial congestion games. B. Durand, W. Thomas, eds. Proc. 23rd Internat. Sympos. Theoretical Aspects of Comput. Sci. Lecture Notes in Computer Science, Vol. 3884. Springer, Berlin, 218-229.

[4] Àlvarez, C., J. Gabarró, M. J. Serna. 2005. Pure Nash equilibria in games with a large number of actions. J. Jedrzejowicz, A. Szepietowski, eds. Proc. 30th Internat. Sympos. Math. Foundations of Comput. Sci. Lecture Notes in Computer Science, Vol. 3618. Springer, Berlin, 95-106.

[5] Awerbuch, B., Y. Azar, A. Epstein. 2005. The price of routing unsplittable flow. Proc. 37th Annual ACM Sympos. Theory Comput., Baltimore, 57-66.

[6] Brandt, F., F. Fischer, M. Holzer. 2007. Symmetries and the complexity of pure Nash equilibrium. W. Thomas, P. Weil, eds. Proc. 24th Internat. Sympos. Theoretical Aspects Comput. Sci. Lecture Notes in Computer Science, Vol. 4393. Springer, Berlin, $212-223$.

[7] Chen, X., X. Deng. 2005. 3-Nash is PPAD-complete. Technical Report 05-134, Electronic Colloquium in Computational Complexity.

[8] Chen, X., X. Deng. 2006. Settling the complexity of two-player Nash equilibrium. Proc. 47th Annual IEEE Sympos. Foundations Comput. Sci., Berkeley, CA, 261-272.

[9] Daskalakis, C., C. H. Papadimitriou. 2005. Three-player games are hard. Technical Report 05-139, Electronic Colloquium in Computational Complexity.

[10] Daskalakis, C., P. W. Goldberg, C. H. Papadimitriou. 2006. The complexity of computing a Nash equilibrium. Proc. 38th Annual ACM Sympos. Theory Comput., Seattle, 71-78.

[11] Dunkel, J., A. S. Schulz. 2006. On the complexity of pure-strategy Nash equilibria in congestion and local-effect games. P. G. Spirakis, M. Mavronicolas, S. C. Kontogiannis, eds. Proc. 2nd Internat. Workshop on Internet and Network Econom. Lecture Notes in Computer Science, Vol. 4286. Springer, Berlin, 62-73.

[12] Fabrikant, A., C. H. Papadimitriou, K. Talwar. 2004. The complexity of pure Nash equilibria. Proc. 36th Annual ACM Sympos. Theory Comput., Chicago, IL, 604-612.

[13] Fischer, F., M. Holzer, S. Katzenbeisser. 2006. The influence of neighbourhood and choice on the complexity of finding pure Nash equilibria. Inform. Processing Lett. 99 239-245.

[14] Fischer, S. T. 1995. A note on the complexity of local search problems. Inform. Processing Lett. 53(2) 69-75.

[15] Fortune, S., J. E. Hopcroft, J. Wyllie. 1980. The directed subgraph homeomorphism problem. Theoret. Comput. Sci. 10(2) 111-121.

[16] Fotakis, D., S. Kontogiannis, P. Spirakis. 2005. Selfish unsplittable flows. Theoret. Comput. Sci. 348(2) 226-239.

[17] Gairing, M., B. Monien, K. Tiemann. 2006. Routing (un-) splittable flow in games with player-specific linear latency functions. M. Bugliesi, B. Preneel, V. Sassone, I. Wegener, eds. Proc. 33rd Internat. Colloquium on Automata, Languages and Programming. Lecture Notes in Computer Science, Vol. 4051. Springer, Berlin, 501-512.

[18] Garey, M. R., D. S. Johnson. 1979. Computers and Intractibility. A Guide to the Theory of NP-Completeness. Freeman and Company, New York.

[19] Goemans, M. X., V. Mirrokni, A. Vetta. 2005. Sink equilibria and convergence. Proc. 46th Annual IEEE Sympos. Foundations Comput. Sci., Pittsburgh, 142-154.

[20] Goldberg, P. W., C. H. Papadimitriou. 2006. Reducibility among equilibrium problems. Proc. 38th Annual ACM Sympos. Theory Comput., Seattle, 61-70.

[21] Gottlob, G., G. Greco, F. Scarcello. 2005. Pure Nash equilibria: Hard and easy games. J. Artificial Intelligence Res. 24 357-406.

[22] Ieong, S., R. McGrew, E. Nudelman, Y. Shoham, Q. Sun. 2005. Fast and compact: A simple class of congestion games. Proc. 20th National Conf. Artificial Intelligence and the 17th Innovative Appl. Artificial Intelligence Conf., Pittsburgh, 489-494.

[23] Johnson, D. S., C. H. Papadimitriou, M. Yannakakis. 1988. How easy is local search? J. Comput. System Sci. 37 79-100.

[24] Leyton-Brown, K., M. Tennenholtz. 2003. Local-effect games. Proc. 18th Internat. Joint Conf. Artificial Intelligence, Acapulco, Mexico, 772-780.

[25] Libman, L., A. Orda. 2001. Atomic resource sharing in noncooperative networks. Telecommunication Systems $17385-409$.

[26] Meyers, C. A., A. S. Schulz. 2008. The complexity of congestion games. Working paper, Massachusetts Institute of Technology, Cambridge, MA.

[27] Milchtaich, I. 1996. Congestion games with player-specific payoff functions. Games Econom. Behav. 13 111-124.

[28] Milchtaich, I. 2006. The equilibrium existence problem in finite network congestion games. P. G. Spirakis, M. Mavronicolas, S. C. Kontogiannis, eds. Proc. 2nd Internat. Workshop on Internet and Network Econom. Lecture Notes in Computer Science, Vol. 4286. Springer, Berlin, 87-98.

[29] Monderer, D., L. S. Shapley. 1996. Potential games. Games Econom. Behav. 14 124-143.

[30] Nash, J. F. 1951. Non-cooperative games. Ann. Math. 54 286-295.

[31] Rosenthal, R. W. 1973a. A class of games possessing pure-strategy Nash equilibria. Internat. J. Game Theory 2, 65-67.

[32] Rosenthal, R. W. 1973b. The network equilibrium problem in integers. Networks 3 53-59.

[33] Schäffer, A. A., M. Yannakakis. 1991. Simple local search problems that are hard to solve. SIAM J. Comput. 20 56-87.

[34] Schoenebeck, G., S. P. Vadhan. 2006. The computational complexity of Nash equilibria in concisely represented games. Proc. 7th ACM Conf. Electronic Commerce, Ann Arbor, MI, 270-279.

[35] Shor, M. 2005. Matching pennies. Dictionary of Game Theory Terms, Game Theory .net. http://www.gametheory.net/dictionary/Games/ MatchingPennies.html.

[36] Yannakakis, M. 1997. Computational complexity. E. Aarts, J. K. Lenstra, eds. Local Search in Combinatorial Optimization, Chapter 2. John Wiley and Sons, New York, 19-55. 